\title{
Grass and Gravel: \\ Investigating Visual Properties Preschool Children and Adults Use When Distinguishing Naturalistic Images
}

\author{
Karola Schlegelmilch ${ }^{1}$ and Annie E. Wertz ${ }^{1}$
}

\begin{abstract}
Author Affiliations:
${ }^{1}$ Max Planck Institute for Human Development, Max Planck Research Group Naturalistic Social Cognition, Lentzeallee 94, 14195, Berlin, Germany.
\end{abstract}

\section{Corresponding Author:}

Karola Schlegelmilch, Max Planck Institute for Human Development, Max Planck Research Group Naturalistic Social Cognition, 94 Lentzeallee, 14195 Berlin, Germany.

Email: schlegelmilch@mpib-berlin.mpg.de

\section{Declarations of interest: None \\ Commercial Relationships: None}

\section{Funding:}

This research was supported by funding from the Max Planck Society awarded to A. E. Wertz. During this research, Karola Schlegelmilch was a pre-doctoral fellow of the International Max Planck Research School on the Life Course (LIFE, www.imprslife.mpg.de; participating institutions: Max Planck Institute for Human Development, Freie Universität Berlin, Humboldt-Universität zu Berlin, University of Michigan, University of Virginia, University of Zurich).

Web link to data:

https://osf.io/8xy5n/?view_only=6ddced286c31456fae7d20dd86e072e6 


\begin{abstract}
Little is known about young children's ability to visually categorize complex structures of a naturalistic environment. To investigate this, we analyzed the relevance of different visual properties and superordinate category information to 4 - to 5-year-old children's $(\mathrm{N}=$ 76) and adults' $(\mathrm{N}=72)$ card sorting decisions. Participants sorted cards depicting complex greyscale images of manmade artifacts, non-living natural elements (e.g., stones), and vegetation into groups, first according to visual similarity and then by the images' superordinate categories. We found that visual properties defined by characteristics of higherorder computations (e.g., variations in shape, distributed detail) predicted children's categorization less than adults. Both age groups' similarity judgements were influenced by the images' assigned superordinate categories — especially by vegetation. Children discriminated vegetation images better than adults when controlling for their overall lower performance. Taken together, these findings demonstrate children's adaptive and flexible processing strategies for complex naturalistic images.
\end{abstract}

Keywords: categorization; perceptual development; image statistics; naturalistic images 


\section{Highlights}

- Preschoolers readily categorized complex naturalistic structures.

- Semantic and perceptual characteristics predicted children's similarity judgments.

- Children flexibly integrated visual properties from different visual hierarchies.

- Shape elements predicted children's categorization less than holistic properties.

- Actively explorable properties played a special role in children's categorization. 


\section{Introduction}

Our daily environment consists of assemblies of complex structures, for example rocks surrounded by shrubbery, or the differently patterned clothes piled up in our wardrobe. Adults are adept at distinguishing these entities visually, even if the visual information is reduced to two dimensions and color cues are lacking, like on a photograph (Renninger \& Malik, 2004). However, for young children this might be a more laborious task, particularly if image regions only vary in characteristics of their visual structure. Some fine-grained or complex visual information of diagnostic properties might exceed the child's developing visual abilities (e.g., Kellman \& Arterberry, 2007; Siu \& Murphy, 2018), making it difficult to distinguish the depicted entities. Previous research shows that, starting in infancy, young children are sensitive to visual signals that enable them to perceive relevant properties of their environment, such as changes in contrast, orientation, or size, and also some higher order properties such as visual configurations (e.g., as in faces), or pictorial depth cues (e.g., Aslin \& Smith, 1988; Braddick \& Atkinson, 2011; Daw, 2014; Kellman \& Arterberry, 2007). Yet, lower order visual abilities such as spatial acuity and contrast sensitivity do not reach adult levels before late childhood (Almoqbel et al., 2017; Ellemberg et al., 1999; Leat et al., 2009). The ability to perceive detailed spatial relationships (e.g., vernier acuity) continues to develop even into the teenage years (Bondarko \& Semenov, 2012; Dekker et al., 2019). When images depict scenes with overlapping and heterogeneous elements - such as naturalistic scenes like rocks surrounded by shrubbery - the perception of differences between the small elements' shapes and arrangement within the visual structures is essential for organizing the scenes into meaningful areas. Visual mechanisms involved in perceptual organization include higher order abilities such as texture segregation (the effortless discrimination of texture features; Kastner et al., 2000; Landy \& Graham, 2004) and contour grouping (e.g., Elder \& Goldberg, 2002; Geisler et al., 2001). Thus, the extraction of spatial characteristics and the structure elements' shape are prerequisites for successful identification (Panis et al., 2008; Perrinet \& Bednar, 2015). These higher order abilities were found to mature around the age of 13 years (for a review see: Taylor et al., 2014). 
CHILDREN AND ADULTS, DISTINGUISHING NATURALISTIC IMAGES

Adults perceive cluttered natural scenes with little effort, suggesting that the adult visual system is shaped phylogenetically and ontogenetically by the tasks and physical properties inherent in human environments (Field, 1987; Geisler, 2008; Kayser et al., 2004; Nassi \& Callaway, 2009; Shepard, 1992). Regularities inherent in visual stimuli serve as cues for visual categorization (Geisler, 2008; Torralba \& Oliva, 2003). From their first months of life, infants build up a basis for perceptual organization and visual categorization (Bhatt \& Quinn, 2011; Goldstone, 1998). The ability to differentiate variations and combinations of visual properties increases during development, continuing into adulthood, leading to more versatile distinctions between object categories or single percepts (Goldstone, 2003; Smith, 1979), and they increasingly process frequently occurring combinations of stimuli as one unit (Goldstone, 1998). The visual system is most susceptible to experience-driven adaptations and refinement up until early adolescence (Fantz \& Nevis, 1967; Maurer \& Lewis, 2013; Siu $\&$ Murphy, 2018). For a younger child, therefore, some specific differences in visual regularities may be imperceptible.

Studies investigating early categorization abilities frequently adapt their stimuli to young children's visual abilities by selecting artificial or graphically simplified stimuli (e.g., Badger \& Shapiro, 2012; Gelman \& Markman, 1987; Inagaki \& Hatano, 1996) or by extracting object stimuli from their background (e.g., Emberson \& Rubinstein, 2016; Hurley et al., 2010; Jeschonek et al., 2010). However, research with artificial or bounded stimuli lacks important aspects of visual categorization, such as visual processing steps related to perceptual organization (Kellman, 2001). Then again, visual processing advantages in young children that are related to the adaptation of the human visual system towards environmental visual tasks (Kayser et al., 2004) would more likely be revealed by naturalistic stimuli. However, such research is still rare. The existing studies conducted with more naturalistic stimuli have targeted, for example, the effect of low-level saliency on the detection of faces (Amso et al., 2014; Frank et al., 2012), the predictability of gaze patterns to features of a visual scene beyond low-level salience (Pomaranski et al., 2021), and perceptual adaptation to visual properties in materials (Balas, 2017; Balas, Auen, Thrash, et al., 2020) or natural scenes (Ellemberg et al., 2012). The visual properties these studies investigated include global vs. local features (Balas et al., 2020), summary statistics (i.e., an algorithm by: Portilla \& Simoncelli, 2000; included in: Balas et al., 2018; Balas \& Saville, 2021; Balas \& Woods, 2014), attention to social signals (Amso et al., 2014; Frank et al., 2012; Pomaranski et al., 
2021), and the distribution of spatial scales (Ellemberg et al., 2012). However, thus far, there is little conclusive evidence that young children show similar processing advantages for image properties that are part of naturalistic scenes as adults. Nevertheless, this research indicates that (i) sensitivity to naturally appearing visual regularities, and (ii) sensitivity to entities with ecological significance (e.g., faces, natural scenes) affected processing of realworld images in young children.

The current study was designed to explore the influence of these two factors on categorization development by comparing preschool children's and adults' performance in card-sorting tasks with images depicting real-world structures. The tasks included two distinct notions of categorization: judgments of perceptual similarity between images, and classification of images to one of three superordinate categories. We also had assessed a set of visual properties from the images. Participants' categorization decisions were then related to the visual properties and superordinate categories. By comparing the predictive value of the visual properties and superordinate categories between the participant groups, we expected to gain insights into children's particular sensitivities to the visual environment.

In the following, we will introduce the theoretical context and the rationale behind the choice of categories and visual properties.

\section{The Development of Visual Categorization}

Perceptual similarities between stimuli elicit category formation and induction in infancy and early childhood (Badger \& Shapiro, 2012; Madole \& Oakes, 1999; Sloutsky \& Fisher, 2004). Infants and young children incidentally form categories when they are exposed to stimuli that have correlating properties (e.g., Rakison \& Yermolayeva, 2010; Younger \& Gotlieb, 1988). With increasing age and experience during development, categorical differentiations become more fine-grained (e.g., Fisher et al., 2015; Smith, 1979), and more distinctions and similarities among entities are noticed, motivating an organization of categories into hierarchies in which more specific classes are included in more general ones (Markman, 1989). Social interactions also affect the way in which a young child's attention is directed at certain visual characteristics (e.g., Köster et al., 2017), and in providing additional cues that support category formation, such as language (Markman, 1989; Nazzi \& Gopnik, 2001; Westermann \& Mareschal, 2012). 
Some research emphasizes the relevance that more general superordinate categories such as plants, non-living natural entities, human-made objects or animals have to humans (e.g., Carrara \& Mingardo, 2013; Gelman, 1988; Opfer \& Gelman, 2011; Wertz, 2019). Foundationally different statuses are given to these categories. For example, some categories (e.g., animals) are viewed as more natural whereas others are seen as being grounded on conventions (e.g., artifacts; Rhodes \& Gelman, 2009). However, multiple sources of information are likely to contribute to the formation of perceptually heterogeneous superordinate categories (e.g., vegetation, artifacts), some of which refer to their function or behavior (e.g., Casler \& Kelemen, 2005; Opfer, 2002). The distinction of living and nonliving things (e.g., by drawing upon their goal orienting behavior; Opfer, 2002) emerges during infancy (Rakison \& Poulin-Dubois, 2001), but is found to develop still during school age. Such additional sources of information underlying category formation are an important part of the concepts behind more general classes of entities and represent important aspects of the significance these categories have for humans.

Researchers have proposed that humans are equipped with specific sensitivities to certain categories due to their relevance over evolutionary time, and are prepared to process information about those categories in ways that allow for rapid responses and efficient learning (e.g., H. C. Barrett, 2014; New et al., 2007; Pauen \& Hoehl, 2015). Visual sensitivity can enhance learning and facilitate adaptive responses in infancy and early childhood (LoBue \& Adolph, 2019; Rakison \& Poulin-Dubois, 2001). For example, research has shown that infants and young children rapidly orient towards threatening stimuli presented as images of ancestrally relevant threats like spiders and snakes (LoBue \& DeLoache, 2008; LoBue \& Rakison, 2013; Rakison \& Derringer, 2008), and young children show specialized reasoning towards artifacts (e.g., Casler \& Kelemen, 2005). Possibly, such sensitivities also support the ability to visually categorize ecologically significant superordinate categories.

We chose vegetation as one of the categories included in the current project. Plants have posed significant benefits and costs for humans over evolutionary time (see e.g., Wertz, 2019). However, visual characteristics of plants are variable and complex. They include visual properties such as symmetry, occlusion, and the repetition of parts varying in their orientation and size, for which young children were found to possess limited perceptual abilities (e.g., Bornstein \& Stiles-Davis, 1984; Siu \& Murphy, 2018). Nevertheless, infants 
already respond differently to plants than manmade artifacts, animals, and other natural kinds (e.g., rocks, shells; Elsner \& Wertz, 2019; Mandler \& McDonough, 1998; Wertz \& Wynn, 2014a) and selectively learn about plant properties such as edibility (Wertz \& Wynn, 2014b, 2019). Although plants are not explicitly acknowledged as living things until later in childhood (Carey, 1988; Opfer \& Gelman, 2011), young children possess rich representations of plants. They understand that plants can grow, need water and sunshine to do so, and can die (Backscheider et al., 1993; Inagaki \& Hatano, 1996; Nguyen \& Gelman, 2002).

As contrasting categories, we chose artifacts and non-living natural elements. Artifacts in the form of tools, articles of daily use, and architecture dominate most living spaces of modern industrialized cultures. Artifacts became increasingly manufactured and artificial only in more recent history (e.g., Read \& Van Der Leeuw, 2008). At a basic level, artifacts differ from naturally-occurring entities like vegetation in that they are intentionally created or selected to be used (Carrara \& Mingardo, 2013). Of course, artifacts are often made from vegetation and other naturally-occurring entities (e.g., Hardy \& Martens, 2016), so the distinction applies to finished artifacts. Thus, the categorization of artifacts is special in that it depends on a given context, intention, or purpose (Keil et al., 2007; Malt \& Sloman, 2007). Accordingly, preschool children were found to learn to group artifact objects according to their function or the way they are used (e.g., Casler \& Kelemen, 2005; Matan \& Carey, 2001; Truxaw et al., 2006).

In comparison, the non-living natural elements in the third category have been part of the human environment as long as vegetation. This category covers the physical components and substances of the natural world (e.g., water, minerals). Their particular occurrence in the environment in the form of lakes, soil, rocks, or snow is a foundation for the existence of living things (e.g., Brant, 1987), and it affected the design and usage of the environment by humans (Behrensmeyer, 1982). On a large scale, and similar to vegetation, natural elements provide a visual background, and can play a role as landmarks in navigation Furthermore, in interactions with the natural environment, contact with natural elements frequently occurs through moving on or in them. Their physical characteristics are learned during everyday actions (like the peculiarities of locomotion on uneven rocks, ice, or in water; see also: Pelz \& Rothkopf, 2007), whereas a precise classification of exemplars or instances of natural elements is not as essential (Hoffman \& Singh, 2012) as it is in plants (e.g., for food selection; 
CHILDREN AND ADULTS, DISTINGUISHING NATURALISTIC IMAGES

Hardy, 2018; Şerban et al., 2008; Wertz, 2019). Developmental research on the categorization of non-living natural elements is still rare (on processed or natural materials see e.g., Balas, 2017; on substances see e.g., Hespos et al., 2016).

\section{Visual Properties of Naturalistic Superordinate Categories}

Although the visual categorization of perceptually heterogeneous higher-order categories is as much a subject in research with adults as it is with young children (e.g., Jozwik et al., 2016; Warrington \& McCarthy, 1987; Zachariou et al., 2018), most of its insights refer to the adult visual system. In visual classification tasks, adults' performance is predicted by properties that are processed at all levels of the visual stream, ranging from low level statistics and texture characteristics to higher order visual information (e.g., Nosofsky et al., 2017; Schmidt et al., 2017). For the current investigation, we considered candidate visual properties which have been found to differentiate natural scenes, textures, or superordinate categories. In preparation for the study, we reduced a more extensive list of visual properties selected from the literature on visual categorization (e.g., Baumgartner et al., 2013; Geisler, 2008; Heaps \& Handel, 1999; Isherwood et al., 2017; Schmidt et al., 2017) and computer vision (Clausi, 2002; Costa et al., 2012) to receive a set that covered diverse qualities and referred to substantial visual tasks related to the human environment (see Table 1; for more information on the selection process see Supplementary Information (SI), A.3 and Figures A.1-2).

\section{Table 1}

Definitions of the Visual Properties

\begin{tabular}{lll}
\hline Name & Definition & Relevance \\
\hline & & Computational $^{\text {a }}$ \\
\cline { 2 - 3 } Alpha & $\begin{array}{l}\text { Steepness of the distribution of energy } \\
\text { across spatial frequencies (SF) }\end{array}$ & $\begin{array}{l}\text { In natural scenes, alpha values are found to lie } \\
\text { in a typical range. The adult visual cortex is }\end{array}$ \\
& $\begin{array}{l}\text { larger changes to more narrow changes } \\
\text { between light and dark image regions. }\end{array}$ & Hansen \& Hess, 2006).
\end{tabular}


Deviation Deviation (i.e., area under the curve) of Deviation differs between artifacts, plants, and an image's actual SF distribution from natural scenes (Redies et al., 2007). In the line fitted to this distribution naturalistic scenes, low values of deviation defined by Alpha (Redies et al., 2007). relate to higher scaling-invariance (e.g., Burton Deviation distinguishes images in \& Moorhead, 1987), in that movement towards which some SF dominate from images the scene does not change its SF-distribution. with more evenly distributed SF.

CooCor Co-occurrence Correlation. Repeating probabilities of neighboring pixels over the image dimensions (Haralick et al., 1973). Sensitive for low-level irregularities of naturalistic textures.

Skew

Skew of the pixel luminance histogram. Related to impressions of shade, lighting, and the greyscale images' original colors (e.g., Graham et al., 2016; Kim \& Anderson, 2010).

Rated $^{\mathrm{b}}$

Curvature Angularvs. curved.

Perceived curvature supports classification between animate and inanimate objects (Long et al., 2017; Schmidt et al., 2017).

Depth Plane vs. three-dimensional.

Indicates spatial arrangement of scene elements. Significant for scene segmentation and action planning (e.g., Atkinson \& Braddick, 2013)

Gloss Dull vs. reflecting.

Surface property, supports classification of materials (Fleming, 2017).

Regularity Regular vs. chaotic.

Important characteristic for texture and surface discrimination (Heaps \& Handel, 1999; Rao \& Lohse, 1996).

Size Small vs. large pattern.

The magnitude of elements as depicted on the cards.

Symmetry Symmetrical vs. asymmetrical.

Symmetry attracts attention in natural scenes (e.g., Açık et al., 2009). Characterizes organic or living things (Schmidt et al., 2017).

${ }^{a}$ Computational properties were assessed with functions implemented in Matlab (version R2017b) or provided by literature on image processing (Gonzalez \& Woods, 2018).

${ }^{\mathrm{b}}$ Rated properties were formulated as opposites and judged on a continuous scale by adult participants.

Natural textures are defined as spatially homogeneous, consisting of repeated elements (Julesz, 1981; Portilla \& Simoncelli, 2000; Zhu et al., 2005). They provide important visual information relevant for any level of categorization, such as surface pattern, material properties, or arrangements of things (Balas, 2021) —including the particular way the texture 
elements are organized (e.g., regular vs. chaotic). Early stages of texture processing are fundamental for the segmentation of visual scenes (Marr, 1976). Because these early processing levels do not necessarily reach awareness, their characteristics are computationally assessed and based on pixel luminance levels of digitized images. Such computational approaches capture characteristics of the luminance histogram and spatial frequency information, but can also lead to complex parametric texture models (e.g., Portilla \& Simoncelli, 2000; Wallis et al., 2017; Zujovic et al., 2013). In order to increase the differentiability between particular texture properties, we have decided against the model of texture analysis by Portilla and Simoncelly (2000) — in spite of its frequent inclusion in developmental studies (e.g., Balas et al., 2018; Balas \& Saville, 2021). This model also fails to represent some characteristics of the current image structures (e.g., long range contours, contrasts between straight and curved lines, assemblies of identifiable elements; Portilla \& Simoncelli, 2000, p. 63), which could bias the differentiation of categories (e.g., artifacts vs. natural elements) in our study. It has been shown that less complex image statistics that assess distribution, contrast, or structure of pixel luminance values can provide diagnostic visual information (Baumgartner \& Gegenfurtner, 2016; Clausi, 2002; Geisler, 2008), and we included such pixel statistics of visual pattern here (i.e., the properties termed CooCor, Skew; see Table 1)

Spatial frequencies quantify the changes between light and dark image regions of different size and orientation. Visual scenes depicting natural environments usually have a fractal-like quality, meaning that their spatial frequency distribution stays approximately the same even if one zooms into the image (termed scale-invariance; Burton \& Moorhead, 1987; Knill et al., 1990; Ruderman, 1997). The slope of a line fitted to this distribution is represented by the value of alpha (see Figure 1 for examples). Scaling invariance and steepness of spatial frequency distributions are important statistics investigated in the context of visual adaptation to the natural environment. Images within certain ranges of alpha are found to be more visually discriminable than images with alpha values outside these ranges (Hansen \& Hess, 2006; Isherwood et al., 2017). Alpha has also been found to differ between categories of image content (Redies et al., 2007), see alpha, deviation, Table 1. However, children younger than 10 years of age were not sensitive to typical ranges of alpha (Ellemberg et al., 2012). 

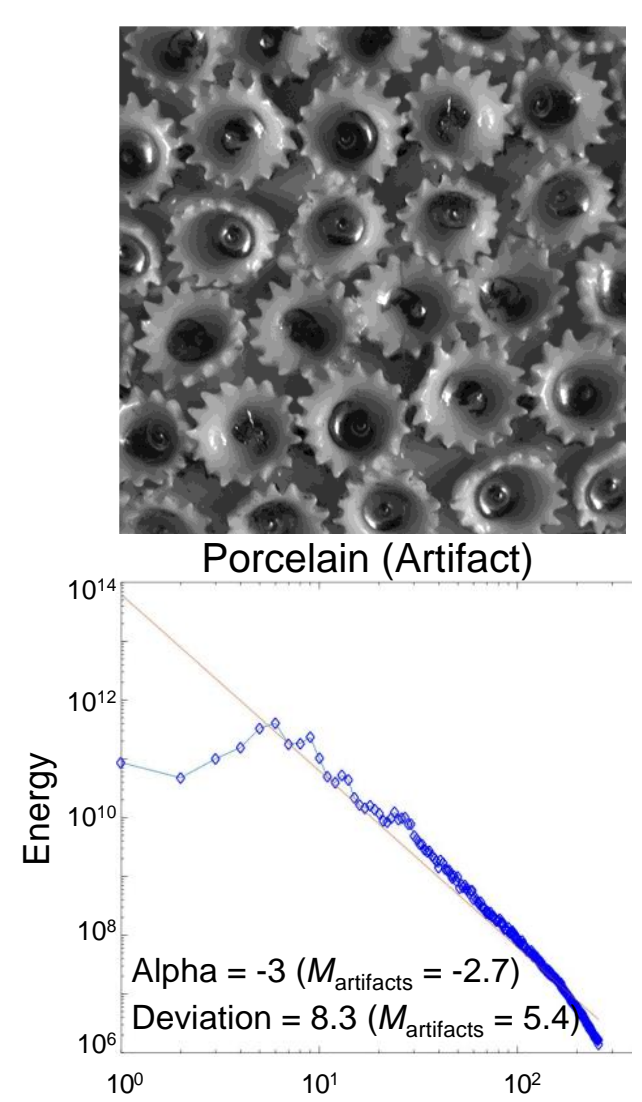

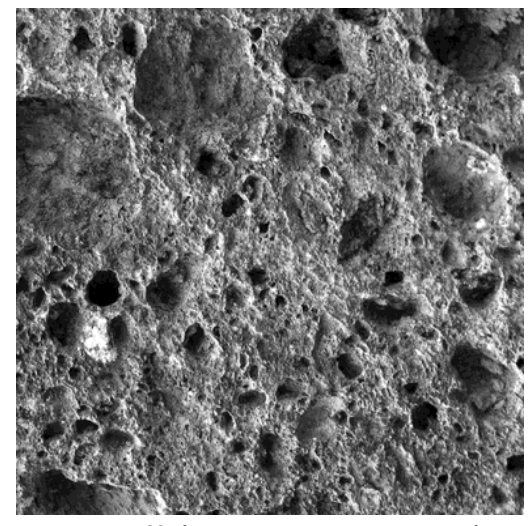

Tuff (Naturel element)

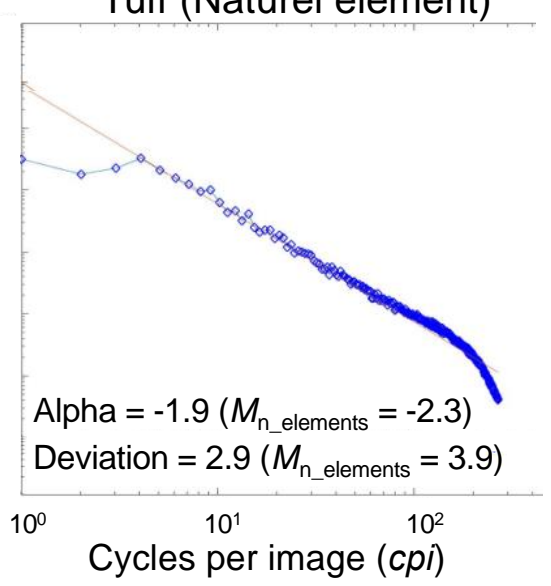

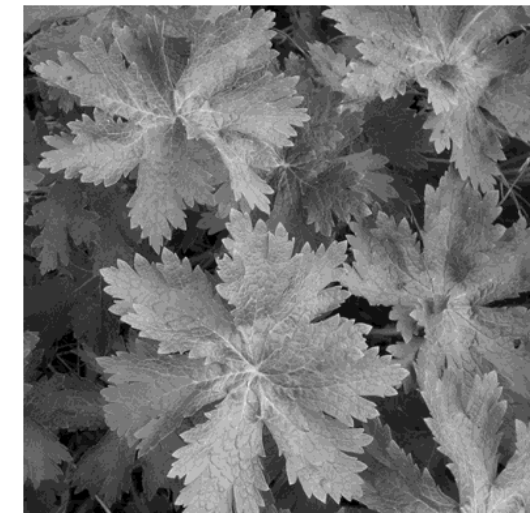

Geranium (Vegetation)

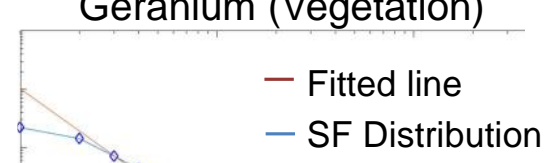

- SF Distribution

Alpha $=-2.4\left(M_{\text {vegetation }}=-2.6\right)$

Deviation $=1.4\left(M_{\text {vegetation }}=4.4\right)$

$10^{\circ} \quad 10^{1} \quad 10^{2}$

\section{Figure 1. Examples of image spatial frequency characteristics.}

Three images taken from the study (top row) and their distribution of spatial frequencies (SF; blue line) plotted on logarithmic scales (bottom row). SF is assessed by Fourier spectral analysis in cycles per image (cpi; alternations between light and dark) plotted on the $x$-axis, with a maximum of $256 \mathrm{cpi}$ for our images analyzed in a resolution of 512 by 512 pixel. Energy refers to the magnitude of SF for all orientations. The fitted line (red) falls with a slope of $1 / f$ alpha. The amount to which the actual distribution of SF deviates from the fitted line (e.g., because some sizes of light-to-dark changes dominate) is quantified by deviation. Porcelain (left) exhibits low alpha, describing a steep fall of the slope, while high Deviation indicates a large variance of SF around the fitted line. The shallower slope of tuff (center) indicates more smaller compared lo large sized SF, and moderate deviation. Geranium (right) has moderate alpha. Its distribution of SF almost overlaps with the fitted line, resulting in low deviation. This indicates that most sizes of light-to-dark changes are evenly distributed in the image, leading to scaling invariance. Values of Alpha and Deviation together with the categories means are given in the Figure below the graphs.

In a behavioral study, Schmidt, Hegele, \& Fleming (2017) compared the classification of unfamiliar objects belonging to three superordinate categories - animals, plants and minerals- to judgments of the objects' properties. Specifically, mid-level shape opposites like 
non-symmetrical/symmetrical, chaotic/regular, or angular/curved increased the probability that an object was assigned to a certain superordinate category. Moreover, material properties can also contribute to superordinate classification. Material properties can be based on subjective experiences induced by movement or touch (e.g., gloss or softness; Fleming, 2017; Hiramatsu et al., 2011), so that studies including these properties frequently rely on human judgments to quantify them (Baumgartner et al., 2013; Fleming et al., 2013; Nosofsky et al., 2017). Similarly, characteristics of the arrangement of small shapes and elements, such as their structural regularity, directionality, depth or roughness (Heaps \& Handel, 1999; Rao \& Lohse, 1993, 1996), can be assessed via human ratings. The visual properties in the current study that were assessed via judgments made by adult raters can be found in the Rated section of Table 1.

\section{The Current Investigation}

In the current study, we investigated preschool children's and adults' categorization of images depicting real-world structures. We used an exploratory approach because developmental research on this topic is still rare. We considered two distinct notions of categorization to uncover determinants of visual categorization: (i) judgments of the visual similarity of structures which does not relate to their identity or class, and (ii) classification, assessed as the assignment of a visual structure to a superordinate category. Judgments of visual similarity can be based on low- and higher-order visual characteristics, including past experiences obtained in contact with the depicted structures (e.g., Tversky, 1977). Judgments of perceived similarity (e.g., via grouping or rating) are a useful categorization technique for visual structures of which labels are missing. Similarity judgments are therefore useful in studies investigating texture perception or unfamiliar objects (e.g., Heaps \& Handel, 1999; Rao \& Lohse, 1996; Schmidt et al., 2017) and are not likely to be affected by differences in the familiarity of entities between children and adults. In contrast, classification performance is affected by the familiarity of the particular entities. Nevertheless, individuals generalize class membership to novel instances on the basis of some internalized visual representation of a superordinate category (e.g., Quinn, 2011). Thus, classification tasks rely to some extent on inferences based on the appearance of the to-be-classified visual structure. Using a 
combination of similarity judgments and classification performance therefore allows us to assess an individual's sensitivity to visual properties and image content.

In the current study, participants grouped one set of images according to similarity, and inferred the superordinate category membership of another set of images. The images in both sets belonged to one of three superordinate categories - artifacts, non-living natural elements, and vegetation. We evaluated the performance of children and adults in each of the tasks, assessed the predictive value of the visual properties for the categorization decisions in both tasks, and, in addition, the predictive value of the assigned categories (i.e., categories assigned to the images in the classification task) for the similarity judgments. We utilized this multi-method approach, because it could best serve the exploratory nature of the present study (studies using a similar multi-method approach: e.g., Gegenfurtner \& Rieger, 2000; Heaps \& Handel, 1999; Nosofsky et al., 2018; Rao \& Lohse, 1993). Additionally, this approach has the advantage of being appropriate for use with both children and adults (e.g., Sloutsky, 2003).

Despite the exploratory nature of the study, we expected that young children would categorize images depicting visual structures of their environment differently than adults. The literature on visual development suggests that visual categorization of naturalistic visual structures requires significant processing effort in young children due to characteristics such as fine details, variations in the shape of repeated elements, or complex arrangements of scene components (Ellemberg et al., 1999; Kimchi, 2015; Siu \& Murphy, 2018). Therefore, children may be more sensitive to visual properties that require less effort. Additionally, the different role superordinate categories have for the participant groups may also affect categorization (e.g., vegetation as possible food source vs. natural elements as ground for locomotion; Pelz \& Rothkopf, 2007; Wertz, 2019). With the current materials and methods, we aimed to investigate how the image categories and the selected visual properties differed in how they predicted the categorization decisions of the participant groups. We further aimed to examine if differences between preschoolers and adults might be more pronounced in categories with distinct ecological significance, and in visual properties that require more visual processing effort in children. 


\section{Methods}

\section{Participants}

The final sample of child participants recruited from urban and suburban regions of a large European city were 76 preschool children (age in years;months: $M=4 ; 11, S D=0 ; 8$, range $=4 ; 1$ to $6 ; 1,40$ female). One additional child was invited but did not want to participate. Three children participated in the classification task, but did not participate in the sorting task; their data was included in the analysis of the classification task. We chose 4- to 5-year old preschool children because they have already gained substantial experiences of environmental regularities and have more developed concepts of environmental entities and events (Goldstone, 1998; Opfer \& Gelman, 2011), but their visual scene processing is still less affected by the particulars of their culture and language than school-aged children (e.g., Köster et al., 2017; Westermann \& Mareschal, 2012). The adult sample consisted of 72 participants (age: $M=32 ; 11, S D=9 ; 2$, range $=18$ to 55 years; 37 female). All participants had normal or corrected to normal vision. We chose a sample size based on a pilot study which was conducted using tasks similar to those used in the present study. Participants were tested either in a laboratory or in a daycare center. Participants who were invited to the laboratory received 10 Euros while the day-care center received a general donation of toys and books. All children received a participation certificate.

All procedures involving human subjects in this study were approved by the Ethics Committee of the Max Planck Institute for Human Development. Written informed consent was obtained from each adult participant and from a parent or guardian of each child participant before any assessment or data collection. Additionally, all children provided verbal consent before taking part in the tasks.

\section{Stimuli}

The photographs included in the study were taken by the first author using a digital camera (Canon Ixus 85 IS), or downloaded from license-free online repositories (pixabay.com, pxhere.com, gettyimages.de). Selection criteria were that they (1) depicted extracts of real-world structures representing one of the three superordinate categories artifacts, non-living natural elements (e.g., water surfaces or rocks; in the following 
abbreviated to natural elements), or vegetation, and (2) were homogeneous in that each entity was covering the whole image space in a non-manipulated way. We focused on naturalistic structures instead of bounded objects, because they pose everyday visual tasks such as texture segregation and the necessity to alternate between hierarchical levels of structure information. The images were transformed to greyscale using the software Adobe Photoshop (Version 2017.0.0). Although color contributes to the identification of natural entities (Gegenfurtner \& Rieger, 2000), we decided against its inclusion because we expected color to dominate similarity judgments in the sorting task and hide the impact of visual properties that are based on pattern. In our picture set, we adjusted the luminance distribution using the software Adobe Photoshop to reduce the impact of differences in the overall darkness or lightness of the images, by stretching each range of grey-levels to the full range of 1-256. In parallel, we kept the averaged grey close to its original value. This led to grey averages in the range of 71183. The current image set is shown in Figure 2. 
CHILDREN AND ADULTS, DISTINGUISHING NATURALISTIC IMAGES

Artifacts
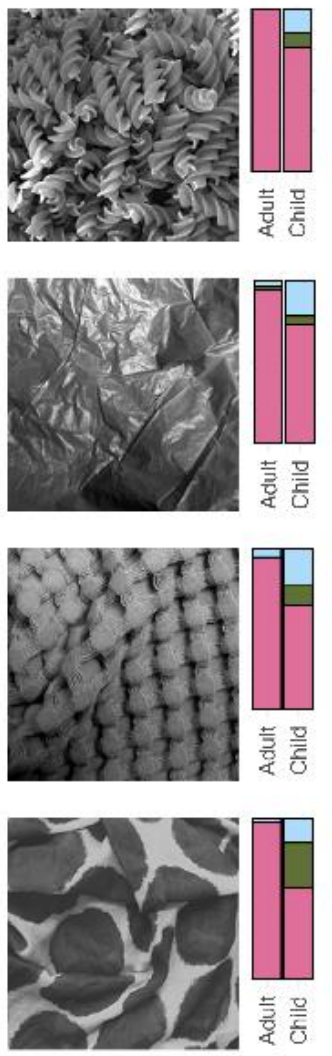

Natural Elements
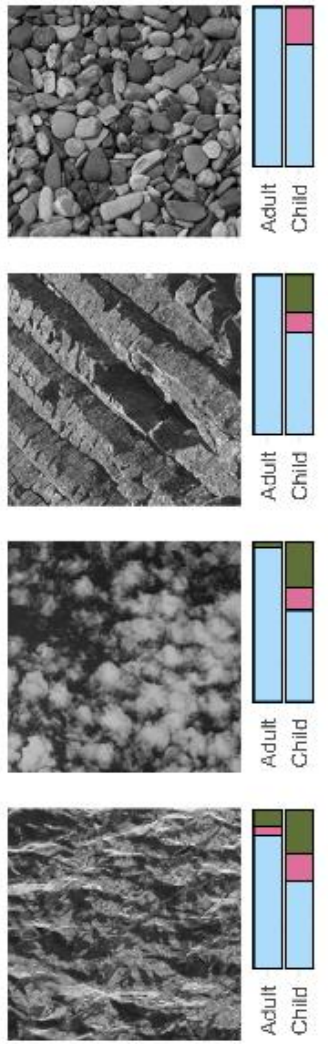
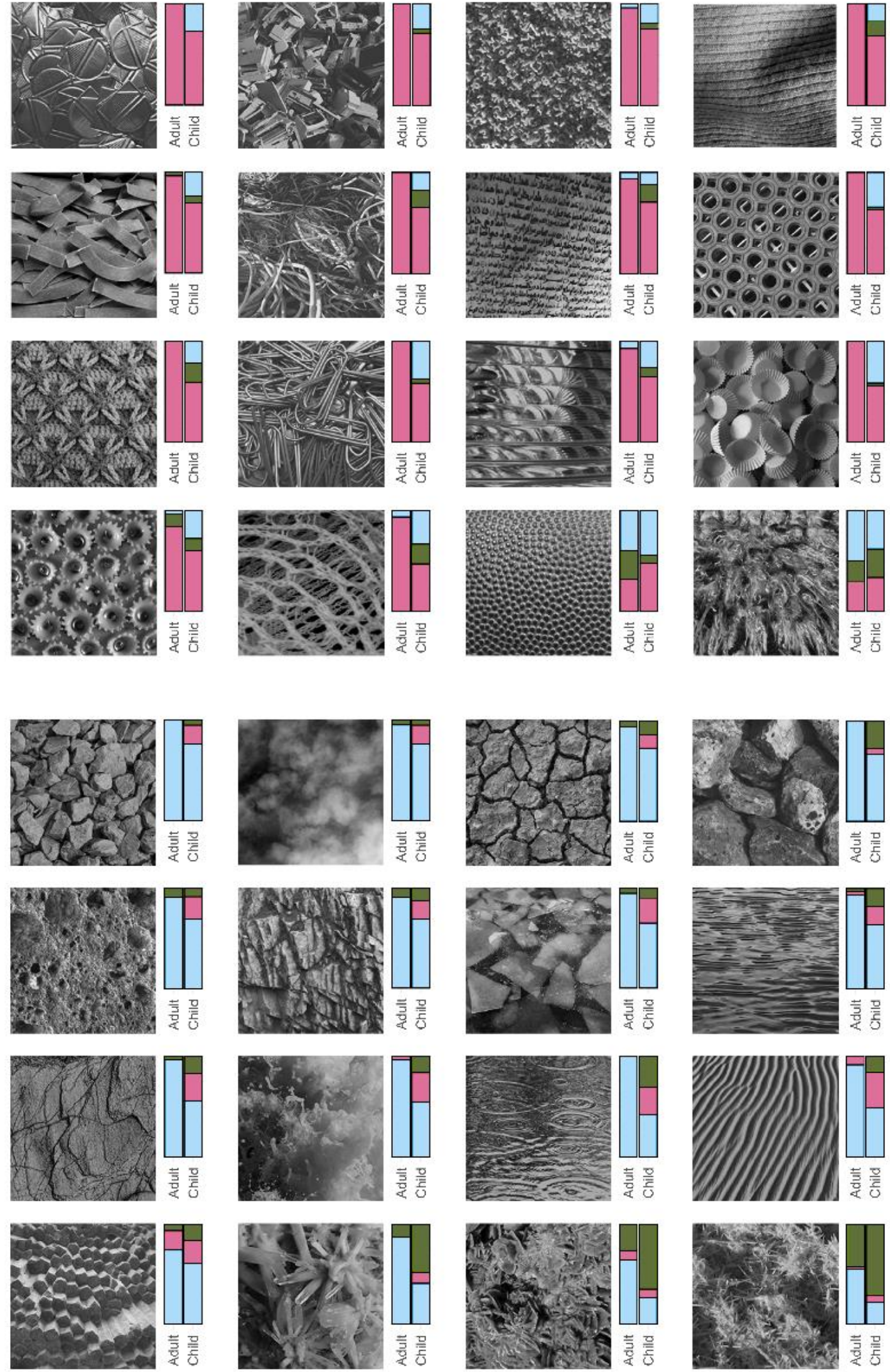

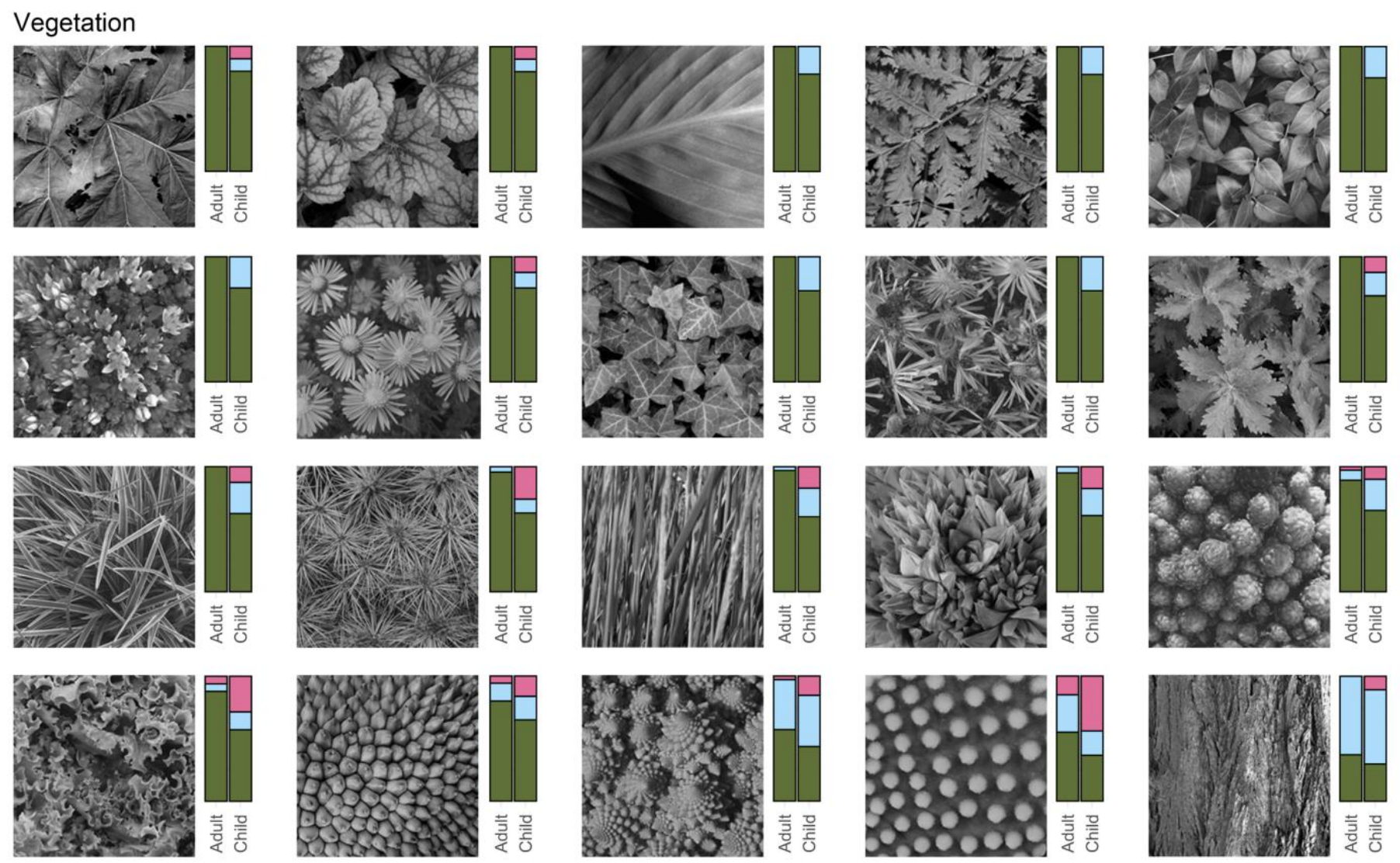

Assigned to: $\square$ Artifact $\square$ Natural element $\square$ Vegetation

Figure 2. Stimulus images and their category assignments by children and adults.

The images of the current study are ordered from top to bottom by their true category, and within each category by the proportion of correct assignments of children and adults in the classification task. Next to each image, colored bars indicate the participant groups' proportional assignments to each of the categories assessed in the classification task. These proportional category assignments were also used in the analysis of the sorting task to evaluate the predictive value of category on similarity judgments.

Classification difficulty was balanced between the categories according to the results of the pilot study in which 26 adults had categorized 141 of our images. We additionally matched the variability of the within-category visual property averages between the categories. With these criteria we aimed to reduce stereotypical representation of the superordinate categories and to produce more perception-related variance (for a similar approach see: Schmidt et al., 2017). The selection process resulted in a final set of 60 images with 20 images per category, shown in Figure 2. The images were then downsized to $1024 \times$ 1024 pixel, printed on card board photo paper and laminated to obtain sorting cards of size 


\section{CHILDREN AND ADULTS, DISTINGUISHING NATURALISTIC IMAGES}

$9.7 \times 9.7 \mathrm{~cm}$. The square format was chosen to reduce biases of orientation and gravity. These cards were split into two halves so that different images could be used in the two categorization tasks, respectively.

\section{Procedure}

Each participant completed a sorting task and a classification task. The stimuli and tasks were identical for children and adults except that children were told cover stories around the instructions to make the tasks more child-friendly. We selected 60 images to use in these tasks in order to obtain as many exemplars of each category as possible and account for the limited attention span of preschoolers, as confirmed by a pilot study with preschoolers. Before each session the full set was separated into two halves of 30 cards with equally balanced categories, so that each participant viewed different images during the two tasks. There were 18 different versions - balanced within the participant groups - of how the images were separated into halves. This separation routine provided similar probabilities for each image to be sorted into a group with any other image during the sorting task. In preparation of the classification task, the other half of the images were shuffled to a random order and piled to a stack.

Similarity-sorting task. For both children and adults, the experiment started with a card sorting task in which participants were asked to group one set of 30 images together according to visual similarity. Participants were asked to judge visual similarity subjectively, without taking the identity of the objects depicted on the images into consideration. There were no restrictions on the number of groups or the number of images within each group. For the children's sorting task, the cover story included a bat puppet who liked image patterns but could not sort the cards itself.

At the beginning of the task, nine cards were already placed on the table in a circle to ensure manageable and neutral comparability. Participants were asked to point to a card pair that they perceived to be visually similar. The card pair was put aside and replaced by two new cards by an experimenter. In the following steps, participants could start new card groups, or add single cards to already existing groups. Participants could request additional cards to be put on the table if they did not find a match. This process was repeated until all cards were assembled. If we noticed that participants were only assembling pairs of two cards 
CHILDREN AND ADULTS, DISTINGUISHING NATURALISTIC IMAGES

we reminded them that it was also possible to add cards to these pairs if wished. When a child finished sorting, the bat puppet happily flew over the sorted image groups (see SI, A.1 for the precise instructions of the sorting task).

Directly after similarity-sorting, adult participants received a questionnaire asking about criteria for their similarity judgments. Because we expected that 4- to 5-year-old children might have difficulties with a comparable questionnaire, we instead video-recorded or took notes of children's spontaneous comments on the similarity between images during the sorting task.

Classification task. After a short (approximately 5 minute) break, participants completed the classification task. We utilized a 3-alternative forced-choice paradigm in which participants were seated in front of three boxes representing the categories artifacts, natural elements, and vegetation respectively.

Both participant groups were told that cards with different images would be presented to them. In contrast to the sorting task, we pointed out that it was now important to attend to the things depicted on the cards. Participants were asked to indicate into which of the three boxes they believed a respective card belonged. For children, the categories were represented on the boxes by abstract images with different colors, whereas adults' boxes indicated the category by a label. Definitions of the categories represented by the boxes were then given orally. The wording of these definitions was adapted to be suitable for the different age groups (see Table 2). During the task, children sat next to the experimenter, who showed the stimuli one by one, while adult participants held the image stack by themselves. Participants did not receive feedback regarding the correctness of their decisions, but the children were praised periodically and were reminded to look at the images carefully. If children spontaneously named the depicted entity correctly but could not guess the superordinate category, we assisted by repeating the definition of the correct box and asked if it could fit there. 


\section{Table 2}

Category Definition During the Classification Task.

Category Definition for Adults Definition for Children

\begin{tabular}{|c|c|c|}
\hline Vegetation & $\begin{array}{l}\text { "Cards that depict plants or parts } \\
\text { of plants." }\end{array}$ & $\begin{array}{l}\text { "Anything that you think is a plant or } \\
\text { tree." }\end{array}$ \\
\hline Artifacts & $\begin{array}{l}\text { "Everyday objects and utensils - } \\
\text { everything that you would say } \\
\text { isn't naturally occurring, but has } \\
\text { instead been produced by } \\
\text { people." }\end{array}$ & $\begin{array}{l}\text { "Things that are man-made. You } \\
\text { would know them from seeing them in } \\
\text { your kitchen shelves or in your } \\
\text { bedroom, as parts of houses or on the } \\
\text { street." }\end{array}$ \\
\hline $\begin{array}{l}\text { Natural } \\
\text { Elements }\end{array}$ & $\begin{array}{l}\text { "Things that you would know as } \\
\text { natural materials - that is, things } \\
\text { that you would see in a natural } \\
\text { environment, and which are not } \\
\text { plants, nor animals, nor manmade } \\
\text { objects." }\end{array}$ & $\begin{array}{l}\text { "Natural things - things you might see } \\
\text { in the mountains, or by the sea. } \\
\text { However, plants can't go in there, } \\
\text { since they belong in the first box, and } \\
\text { the same goes for man-made things, } \\
\text { which have their place in the second } \\
\text { box." }\end{array}$ \\
\hline
\end{tabular}

Note: The text provided here is a translation of the original German definitions. For the full German instructions of the classification task and their English translation see SI, A.1, Table A.2.

\section{Data Preparation}

We recorded the images that were assembled within a group by each participant during the sorting task. All possible combinations of image pairs within a group of assembled images were classified as similar to each other, whereas images that were put in different groups, or single images without match, were classified as not similar. We then calculated two proportional similarity matrices with 60 images on the $\mathrm{x}$-and $\mathrm{y}$-axis, separately for children and adults, by dividing the frequencies of similar image pairs by the number of participants who had received the respective images.

In the classification task, we recorded participants' answers for each image, no matter if it was correct or not, leading to the variables Assigned Artifact, Assigned Natural Element, or Assigned Vegetation. 


\section{Results}

Data comparisons in both tasks were conducted as analysis of variance (ANOVA; Rpackage ez; Lawrence, 2016) or generalized linear model (GLM) with the function glm implemented in R (R Core Team, 2019). If models included multiple levels of comparisons (i.e., in the classification task), we conducted generalized linear mixed effect models (GLMM) with the function lmer (R-package lme4; Bates et al., 2015). For GLMs and GLMMs, we defined binomial error structures. Residual and specification diagnostics of the GLMMs were carried out with the R package DHARMa (Hartig, 2020). Influential cases within the units of our comparisons were diagnosed with regard to DFBetas (function influence; R-package lme4), and Cook's D (R-package influence.ME; Nieuwenhuis et al., 2012). In the GLMMs, we assessed the significance of predictors by comparing the current model with a model reduced by the respective predictor with the R-function Anova (package car; Fox \& Weisberg, 2019), which provided $p$-values for fixed effects based on chisquare likelihood-ratio tests. In all comparisons, $p$-values $\leq .05$ were considered as significant.

The data underlying the statistical analysis of this study is accessible under the link https://osf.io/8xy5n/?view_only=6ddced286c31456fae7d20dd86e072e6. We begin by presenting the results of the classification task followed by the results of the sorting task.

\section{Classification Task}

We pursued several goals with the analysis of the classification data. First, we assessed performance measures as a function of participant group (children, adults) and image category (artifact, natural element, vegetation). The rationale for this was to determine the difficulty of the task and sensitivity for the three categories. Then, we assessed the predictive value of visual properties for participant groups' assignments of category membership to the images and compared the predictive value of the visual properties between the participant groups. This was done to investigate if children were more or less sensitive to particular visual properties relative to adults. Finally, we calculated the proportions by which the respective categories were assigned to an image by either participant group. The proportional category assignments - instead of the true categories - were later included in the analysis of 
the sorting task to assess how they predicted similarity judgments between images. We therefore begin by reporting the classification results, even though the classification task was conducted after the sorting task in the experimental procedure in order to minimize an overt effect of category membership on similarity judgments. We first report how the true categories of our image set were predicted by the visual properties, followed by the participants' classification results.

\section{Distribution of the Visual Properties in the Images' True Categories}

We assessed which visual properties statistically predicted category membership in our image set. This allowed us to determine whether respective visual properties had the same predictive value for the true categories in our images, as they did for the participants' assignments of image categories.

For each of the three categories, separate GLMs were conducted on the visual properties of the 60 images used in our study. The binary dependent variables (DV) indicated if an image depicted the respective category or not $(1,0)$. We assessed the significance of visual properties by including each visual property individually in a model, resulting in 10 tests. Figure 3 shows the distribution of visual properties as a function of the categories depicted in the images. Significant main effects are indicated by asterisks. All coefficients and standardized means are provided in Supplementary Tables (ST), Table B.1.

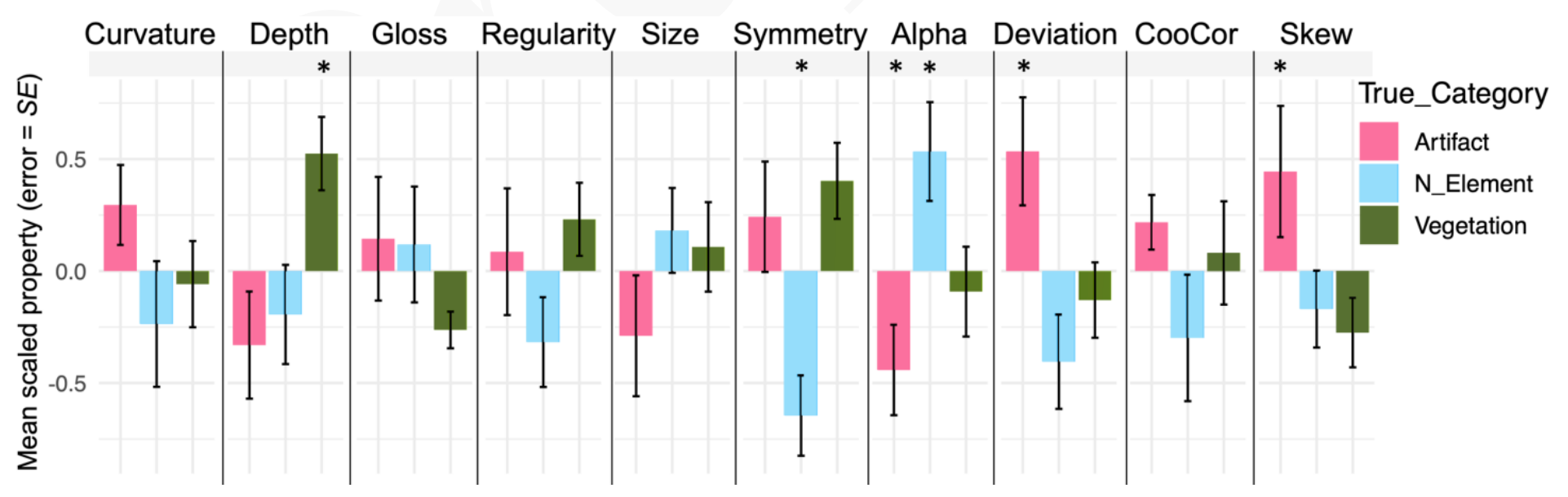

Figure 3. Visual properties as function of the images' categories.

Visual properties are z-standardized within each property and averaged within the respective true categories.

* adjusted $p<.05$ (method: Benjamini \& Hochberg, 1995), assessed in the GLM, see ST, Table B.1. 


\section{Children's and Adult's Classification}

Each of the 76 children who participated in the classification task sorted a complete set of 30 cards into artifact, natural element, and vegetation boxes. Of these cards, children correctly classified on average $69.6 \%$ of the images $(M=20.9, S D=3.8)$. No difference in the proportion of correctly classified images was found between girls $(71.3 \%, S D=11,2 \%)$ and boys $(67.5 \%, S D=14,1 \% ; t=1.3$, n.s.). The continuous variable Age, however, predicted children's proportion of correctly classified images $\left(F(1)=10.8, p=.001, \eta^{2}=.13\right)$. A proportion of $65 \%$ correctly classified images was predicted for the age of 4 years and 4 months (the 5th percentile of the children's age range), while a proportion of $72 \%$ correctly classified images was predicted for the age of 5 years and 10 months (the 95th percentile of the age range), indicating that older children had more correct classifications than younger children. We therefore included the covariate Age in the GLMMs conducted on children's assignments of categories, see SI, A.6.1.

Each of the 72 adult participants sorted a full set of 30 cards into artifact, natural element, and vegetation boxes. Adults correctly classified on average $90.5 \%$ of the sorted images $(M=27.1, S D=1.8)$.

\section{Comparison of Children's and Adult's Classification Results}

The correlation of correctly classified images between children and adults was $r(59)=$ $.74,95 \% C I=[0.59,0.83], p<.001$. Of interest for our study were differences between the participant groups within visual properties or image categories. These differences could specify particular sensitivities underlying visual categorization in children. We therefore conducted comparisons between children and adults which included the factors group (children, adults), category (i.e., the true image categories) and the interaction terms category $\times$ group or visual property $\times$ group. The separate analysis of children's and adults' classification is reported in SI, A.6.

Classification performance. We assessed the discriminability index d-prime ( $d$ '; Wickens, 2002) for each of the true categories, using the R-function dprime (R-package 
psych; Revelle, 2018). Higher values of $d^{\prime}$ indicate a better discriminability of one category from the others (SI, Table A. 3). A $2 \times 3$ ANOVA on the discriminability measure $d^{\prime}$ with the factors category and group confirmed the general difference in discriminability between children and adults with a main effect of group $\left(F(1,146)=164, p<.001, \eta^{2}=.45\right)$, indicating a lower discriminability of the categories in children than adults. Moreover, there was a main effect of category $\left(F(2,292)=33.8, p<.001, \eta^{2}=.055\right)$ and an interaction between group and category $\left(F(2,292)=7.6, p=.001, \eta^{2}=.013\right)$. Post-hoc comparisons (Tukey's HSD) of the main effect of category indicated that within both participant groups, natural elements were classified with lower discriminability than artifacts and vegetation (both $p<.001)$, whereas no difference was found between vegetation and artifacts $(p=.35)$. When comparing the relative discriminability of the categories within children and adults, the group $\times$ category interaction showed that adults' discriminability of vegetation was lower than children's discriminability, $(p=.02)$, indicating that despite children's overall lower ability to discriminate between the categories, they were more likely to discriminate vegetation from the other categories than adults were. No other contrasts were significant.

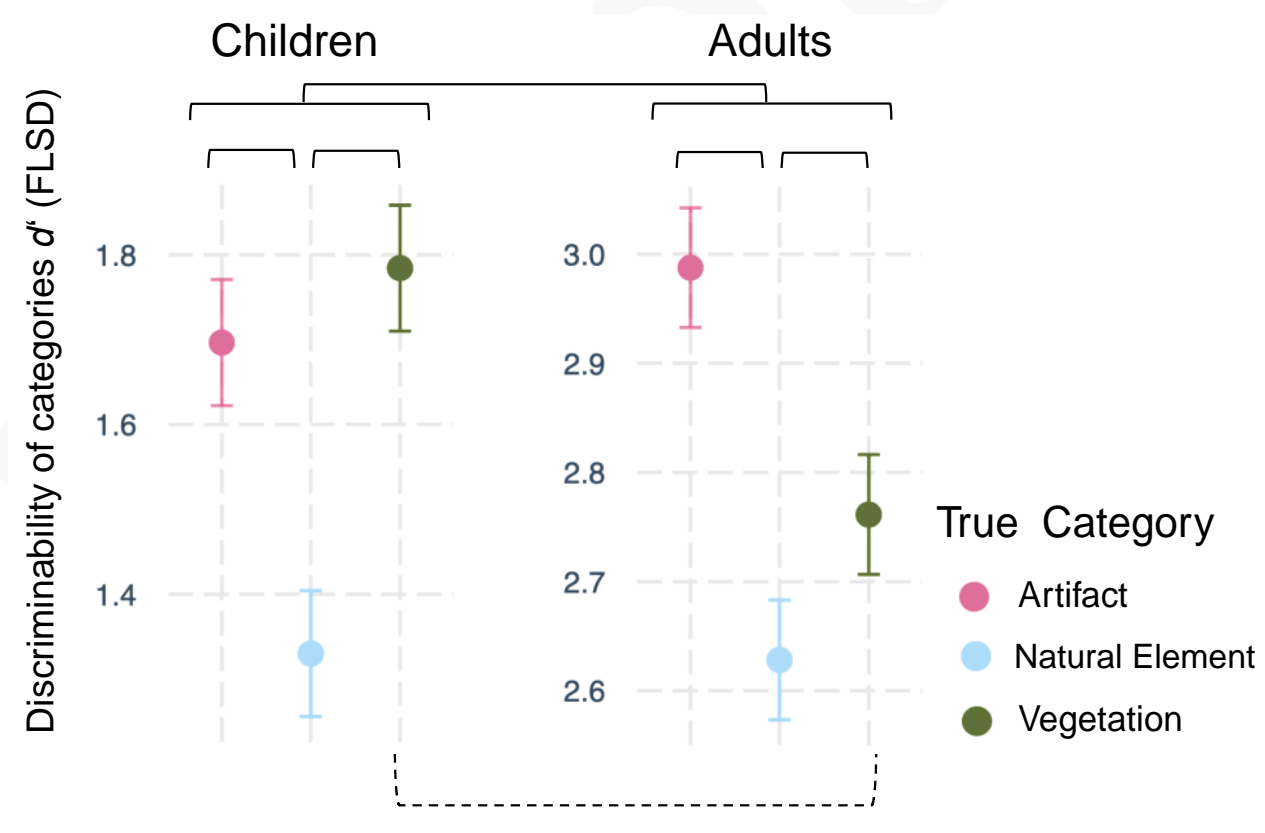

Figure 4. Differences between children's and adults' discriminability of categories.

Predicted scores of $d$ 'indicating the participant groups' ability to discriminate between categories, higher values refer to greater discriminability. Significant effects $(p<0.05)$ are represented by brackets (solid = main effects; dotted = interaction). Note the different levels of the $y$-axes reflecting the main effect between participant groups. 
CHILDREN AND ADULTS, DISTINGUISHING NATURALISTIC IMAGES

Visual Properties Predicting Participants' Category Assignments. In order to extract visual properties that predicted assignments of images to one of the three categories, we conducted GLMMs with a binomial error structure. Participants' responses were binarily coded, resulting in three DVs which indicated if an image was assigned to one of the categories or not $(1,0)$. To account for intra-class correlation, we included participants and images as the units of random intercepts. We assessed the impact of visual properties on category assignment by including each visual property as fixed effect individually in a model, resulting in 10 tests. These tests equivalently explored the predictive value of each property. The reason for choosing separate analysis of visual properties were 1) missing prior expectations about their significances, and 2) the fact that interrelations of simultaneously included IVs in a full model might obscure the impact of some predictors (correlation matrix shown in SI, A.4 Figure A.2). Alternative methods like the agglomeration of properties by PCA might hide their unique contributions, whereas variable selection with specialized methods like lasso regression (Groll \& Tutz, 2014) could generate different results if additional properties than those currently selected were included. These alternative types of analysis would not be as appropriate for the exploratory approach taken here. We controlled the false discovery rate in multiple comparisons by adjusting $p$-values within the 10 tests conducted for each DV using the method of Benjamini and Hochberg (1995). We are reporting the $p$ - values adjusted by this method.

We compared the visual properties of the images that were assigned to the categories by children and adults. GLMMs included the IVs participant group, an individual visual property, the interaction term property $\times$ group, and the random effects image and participant ID. Figure $5 \mathrm{~b}$ indicates visual properties that significantly differed between children's and adults' assignments. Each visual property differed between children and adults in at least one of the three assigned categories. This was most evident in the images assigned to the natural elements category in which all ten properties differed between children and adults. Next was the artifact category in which five properties differed, followed by the plant category in which only three properties differed. Moreover, symmetry and deviation differed between children and adults in all three assigned categories, whereas depth, curvature and size only differed in the natural elements category. Pictorial depth cues predicted children's decisions about category assignments most strongly (Figure 5a). In contrast, alpha — an important predictor of the categories in our image set (Figure 3) — did not contribute significantly to children's 
assignments in any category and differed between children and adults in the artifacts and natural elements categories.

All significant visual property $\times$ group interactions - except deviation on the assigned vegetation category — resulted from a weaker predictive value of the respective property for children's assignments compared to adults. Weaker predictive values are indicated by an average of the scaled visual property that was closer to zero in children than in adults. All adjusted $p<.05$; coefficients are presented in ST, Table B.4.

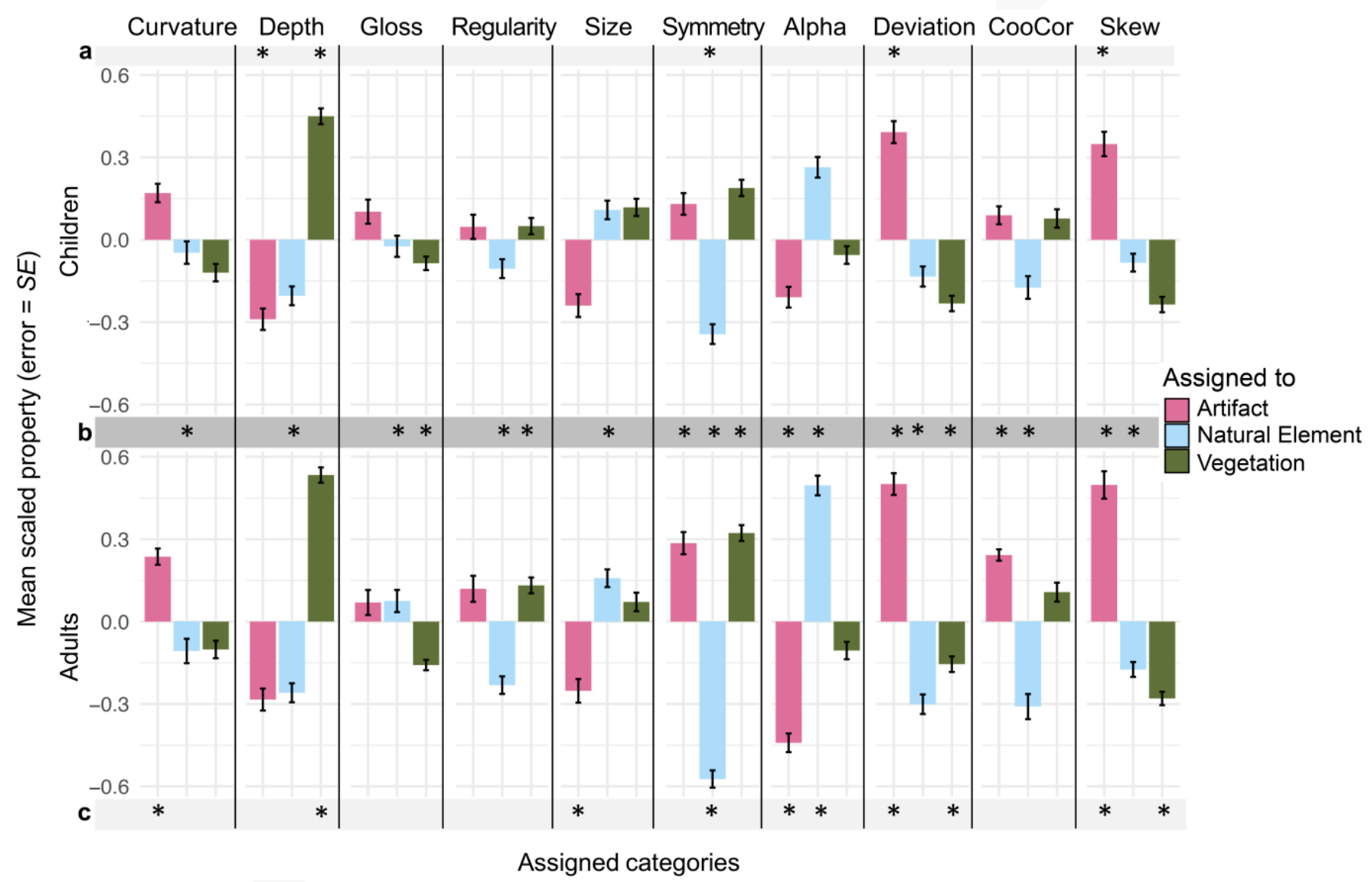

Figure 5. Visual properties as functions of assigned categories by children (top) and adults (bottom).

Properties are z-standardized and averaged over all the images that were assigned to a category by each participant, respectively. Asterisks on dark-grey band in (b) indicate significant interaction terms between participant group and visual property, of the GLMM conducted with the data of both participant groups. Asterisks on light-grey band in (a) and (c) indicate significant main effects of a visual property in the GLMMs conducted on the respective assigned category for children (a) and adults (c), for discussion of group-wise results see SI, A.6. All adjusted $p<.05$ (method: Benjamini and Hochberg,1995). For coefficients and $C /$ values, see ST, Tables B. 2-4. 


\section{Similarity-sorting Task}

For their similarity judgments, participants were asked to attend to the images' visual characteristics apart from the identity of the depicted entities. We aimed to identify which of the current visual properties predicted participants' similarity judgements for naturalistic structures in this task. However, reference characteristics to which similarity is judged without further instruction can vary between individuals (A. Tversky, 1977). If participants base their similarity judgments on specific experiences they have had with a category (e.g., its softness), they might group images that they assume to belong to the same category and have similar properties - independent of the true category of the depicted entities. In the analysis, we therefore also explored whether the similarity judgments were also predicted by the categories that participants had assigned to the images in the classification task (Figure 2).

Similarity judgments can be based on dimensions of image characteristics, in which similarity relies on similar levels within a property's continuum (e.g., Nosofsky et al., 2017; Rao \& Lohse, 1996). Alternatively, similarity judgments can be based on the salience of a set of unique attributes which images share in a context (e.g., Heaps \& Handel, 1999; Tversky, 1977). In order to include these different aspects, we applied the statistical methods hierarchical cluster analysis (HCA; e.g., Friesen et al., 2015; Hair et al., 1998), and nonmetric Multidimensional Scaling (nMDS; e.g., Läge et al., 2011), which allow a dimensional as well as a categorical perspective on multivariate data.

We first conducted HCAs separately for the children's and adults' similarity matrices, using the R-function hclust (R Core Team, 2019) with the Ward2 agglomerative clustering method (Murtagh \& Legendre, 2011; Ward, 1963). HCAs determine a progressive series of more inclusive clusters - starting from unique combinations of image pairs to more general, larger ensembles. Agglomerations of images at a low height (low inter-cluster distance) indicate that these images were judged as more similar than agglomerations at higher stages. The Ward 2 clustering method attempts to merge clusters which lead to a minimum total of within-cluster variance. The groups' HCA solutions were then related to image characteristics (visual properties, assigned categories). HCAs allow us to examine and compare the relationships between similarity judgments and the size of the image groups. By providing the agglomeration process, HCA has an additional dimension that can provide insight into details of the grouping process and structure - in particular, if combined with the visual property 
CHILDREN AND ADULTS, DISTINGUISHING NATURALISTIC IMAGES

data. Next, we conducted nMDS specified for ordinal data with the R-function mds (package smacof; De Leeuw \& Mair, 2011) separately for children and adults. The fit of the nMDS was evaluated according to suggestions of Mair et al. (2016).

\section{Similarity Sorting Performance}

Seventy-three children participated in the sorting task. Seventy children received the full set of 30 images, while 3 children stopped participating after receiving 20, 24 and 24 images, respectively. Children sorted between 15 and 30 images into groups $(M=29.5, S E=$ .24). The remainder of the images were not sorted because the child terminated sorting early, or they were not included into a group because no match was found. In sum, child participants assembled 2153 images. The data of the sorted cards of all children who participated in the sorting task were included in the analysis. On average, each child sorted their images into $M=$ $9.8(S E=0.3$, range $=4-16)$ groups which each included $M=3.2(S E=0.1$, range $=2-8)$ images. This led to 2874 combinations of image pairs that went into the analysis.

Seventy-two adults participated in the sorting task and received the full set of 30 images. Adults sorted between 26 and 30 images into groups $(M=29.3, S E=.12)$, in total 2110 images. The remainder of the images were not sorted into groups because no matches were found. Each adult assembled on average $7(S E=.3$, range $=3-13)$ different groups which included on average $4.7(S E=.2$, range $=2-10)$ images. This led to a total of 4592 paired images.

First, we looked at general differences between the children's and adults' sorting by comparing their agglomeration processes. Children's HCA showed that inter-cluster distances of the children's sorting data ranged from a minimum height of .18 between the two most similar images to a maximum height of 1.89. Adults' inter-cluster distances ranged between close to zero and the maximum of 2.64 (SI, Figure A.4). These differences between the participant groups' inter-cluster distances indicate that children were more flexible in the image groups they assembled, leading generally to less distinct groups (i.e., a lower maximum inter-cluster distance), and more variability in the combinations of individual images (i.e., higher inter-cluster distances between the individual images) than adults. 
CHILDREN AND ADULTS, DISTINGUISHING NATURALISTIC IMAGES

\section{Visual Properties Predicting Children's and Adults' Similarity Judgments}

In order to assess the predictive value of visual properties on the participants sorting decisions, we added the visual property values of the images to the data indicating the images' cluster membership. The impact of a visual property for each step in the clustering hierarchy was assessed by calculating the proportion of variability between the individual clusters to the total variability of the property, specified by $\mathrm{R}^{2}$ (frequently termed "explained variance"; for a similar approach, see: Friesen et al., 2015). Higher levels of the resulting $\mathrm{R}^{2}$ values indicate a stronger variation of the visual property between clusters, interpreted as a stronger predictive value of the property on participants' sorting decisions. The predictive value of assigned categories was assessed with the same procedure.

Evaluation of visual properties' predictive value for the sorting process. In Figure 6 (top row) we plotted the visual properties' $R^{2}$ values as a function of the height of the dendrogram. The top left of Figure 6 illustrates the development of the impact of visual properties on children's sorting decisions, adults' $\mathrm{R}^{2}$ values are plotted on the right.

Visual inspection indicates that in both participant groups, visual properties alternated in their predictive strength depending on the number of clusters in which images were organized, and on the corresponding inter-cluster distance. However, in children, the $\mathrm{R}^{2}$ values of particular visual properties started to vary late at about dendrogram height .4. in contrast, in adults' HCA, visual properties already varied in their impact at minimum dendrogram height, indicating that images that were grouped together by adults frequently corresponded in some of their visual properties.

The predictive values of assigned categories on children's and adults' similarity decisions are depicted in Figure 6 (bottom row). Again, variability between R2 values of the categories increased later in children than in adults. However, the predictive strength of the assigned categories exhibits a similar pattern in children and adults, in that images assigned to the vegetation category were increasingly likely to be assembled than images assigned to the other categories. For all $\mathrm{R}^{2}$ values see online data repository. 


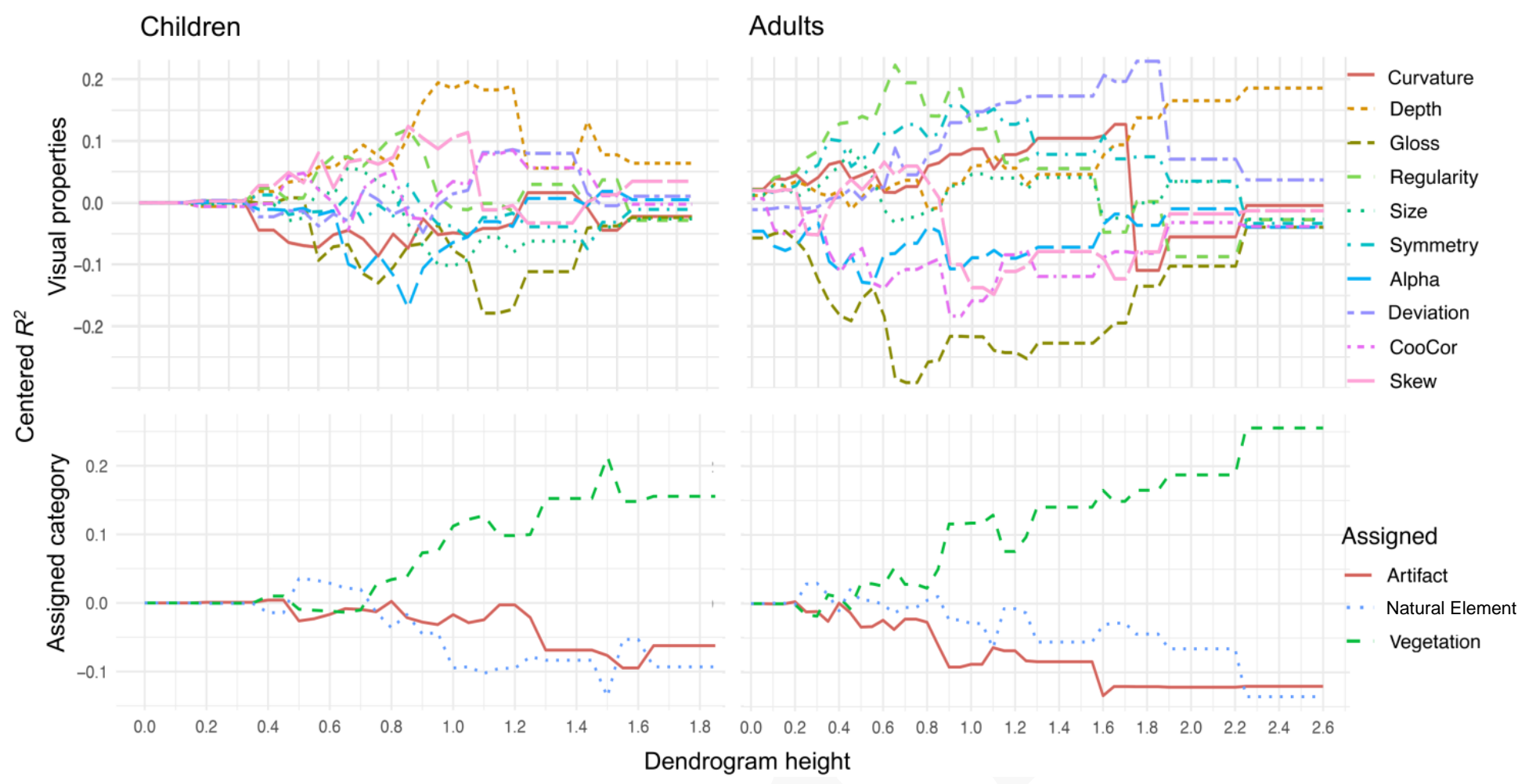

Figure 6. Explained variance of image characteristics by participants' sorting, as a function of the number of clusters.

Levels of $R^{2}$ for image characteristics as a function of the height of the dendrogram, for children (left) and adults (right). Height (x-axis) indicates the inter-cluster distance of images merged within successive hierarchical clusters. $R^{2}$ (y-axis) represents the predictive value of each of the image characteristics on similarity judgments (i.e. explained variance), separately for the 10 visual properties (top row) and for the categories assigned to an image in the classification task (bottom row). $\mathrm{R}^{2}$ was assessed in steps of height .05. For better comparability of the differences between the properties, $R^{2}$ values were centered for each indexed height. A detailed discussion of the predictive value of image characteristics on the sorting judgments (separate and combined participant groups) is provided in the SI, A.7.

\section{Statistical comparison of visual properties predicting children's and adults'}

sorting. To answer the question of whether children and adults generally attended to different visual characteristics in their similarity judgments, we statistically compared their HCA solutions with focus on visual properties and assigned categories. A $10 \times 2$ ANOVA on $\mathrm{R}^{2}$ values of the entire cluster sequence with the factors participant group (children, adults), visual property (10 levels), and their interaction term showed a main effect for participant group $\left(F(1,28)=5.6, p=.025, \eta^{2}=.14\right)$ which was qualified by an overall stronger predictive value of visual properties on adults' similarity judgments. We also found a main 
effect for visual properties $\left(F(9,252)=110, p<.001, \eta^{2}=.38\right)$, and a significant interaction between participant group and visual properties $\left(F(9,252)=51, p<.001, \eta^{2}=.22\right)$. Post hoc comparisons of the interactions revealed that differences between children and adults went in both directions. Deviation, curvature, regularity, size and symmetry predicted children's judgments less than adults' judgments (all $p<.001$ ). In contrast, CooCor and gloss predicted children's judgments to a greater extent than adults' (both $p<.001$ ), while no differences were found for depth $(p=.1)$ and skew ( $p=.921)$, which were strong predictors of children's sorting decisions, and alpha $(p=.372)$, which predicted sorting decisions in both groups to a rather low extent (Figure 7).

A $3 \times 2$ ANOVA on $\mathrm{R}^{2}$ values compared the predictive value of assigned categories on similarity judgments between the participant groups. A main effect for assigned category $\left(F(2,232)=56, p<.001, \eta^{2}=.25\right)$ was qualified by vegetation's stronger predictive value compared to artifacts and naturel elements, which did not differ. The participant groups did not differ $(F(1,116)<1, p=.38)$, and the interaction term did not reach significance $(F(2$, $232)=2, p=.12$ ). These results indicate that similarity judgments were affected by all assigned categories, of which assigned vegetation predicted both children's and adults' similarity judgments most strongly (Figure 7 and SI, A.7. For coefficients and CIs see ST, Tables B.6-11. An analysis of the true categories' predictive values for similarity judgments is given in SI, A.10). 
Visual Properties

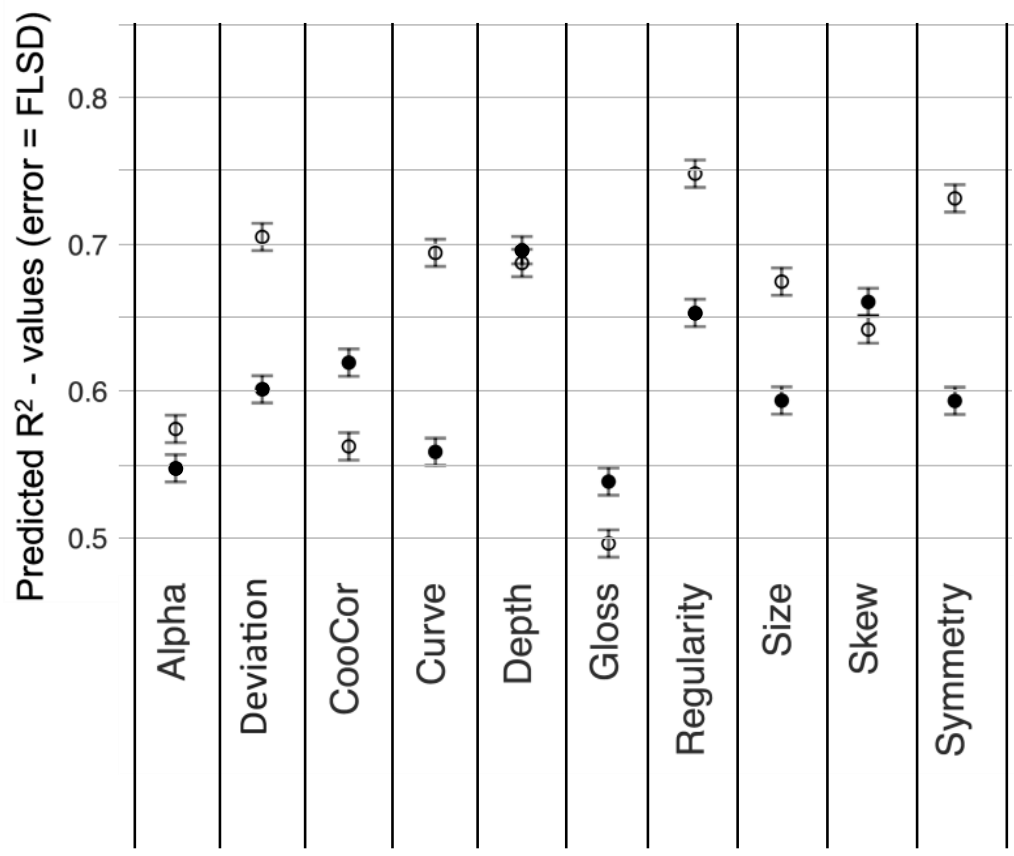

Assigned Categories

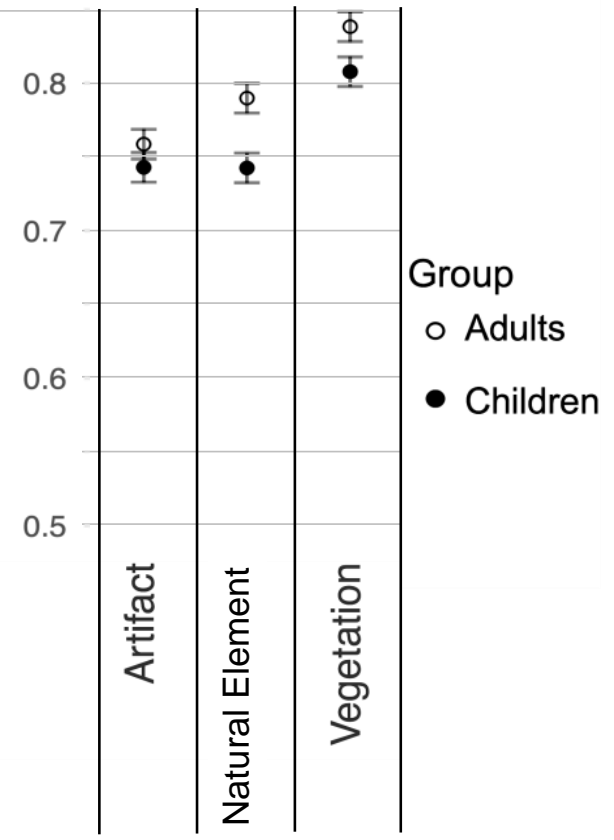

Figure 7. Comparison of $\mathbf{R}^{2}$ values of the similarity-sorting data as functions of visual properties, assigned categories, and participant group.

Predicted means of $\mathrm{R}^{2}$ values in two ANOVAs (visual properties $\times$ group, left, assigned categories $\times$ group, right). $R^{2}$ values (i.e., explained variance) were obtained from the HCA on similarity judgments at each step in the clustering process (60 to 2 clusters). All predicted means and the differences of post-hoc Tukey's HSD comparisons are provided in ST, Tables B.6-11.

\section{Nonmetric Multidimensional Scaling}

Next, we explored how image characteristics explained the way in which participant groups assembled the individual images. In multidimensional scaling, objects are arranged in a multidimensional Euclidian space so that the configuration of the objects represents the distances within the underlying similarity matrix. Including configuration plots of the nMDS solutions in our analysis provides visualization of the similarity judgments related to concrete images. We decided on a two-dimensional nMDS model because it had better clarity and because scree plots did not indicate a more preferable number of dimensions (Mair et al., 2016). 
Figure 8 shows the nMDS configuration plots obtained for children (A) and adults (B). Clusters of images in the nMDS configurations relate to images which were assembled frequently in the sorting task due to their perceived similarity.

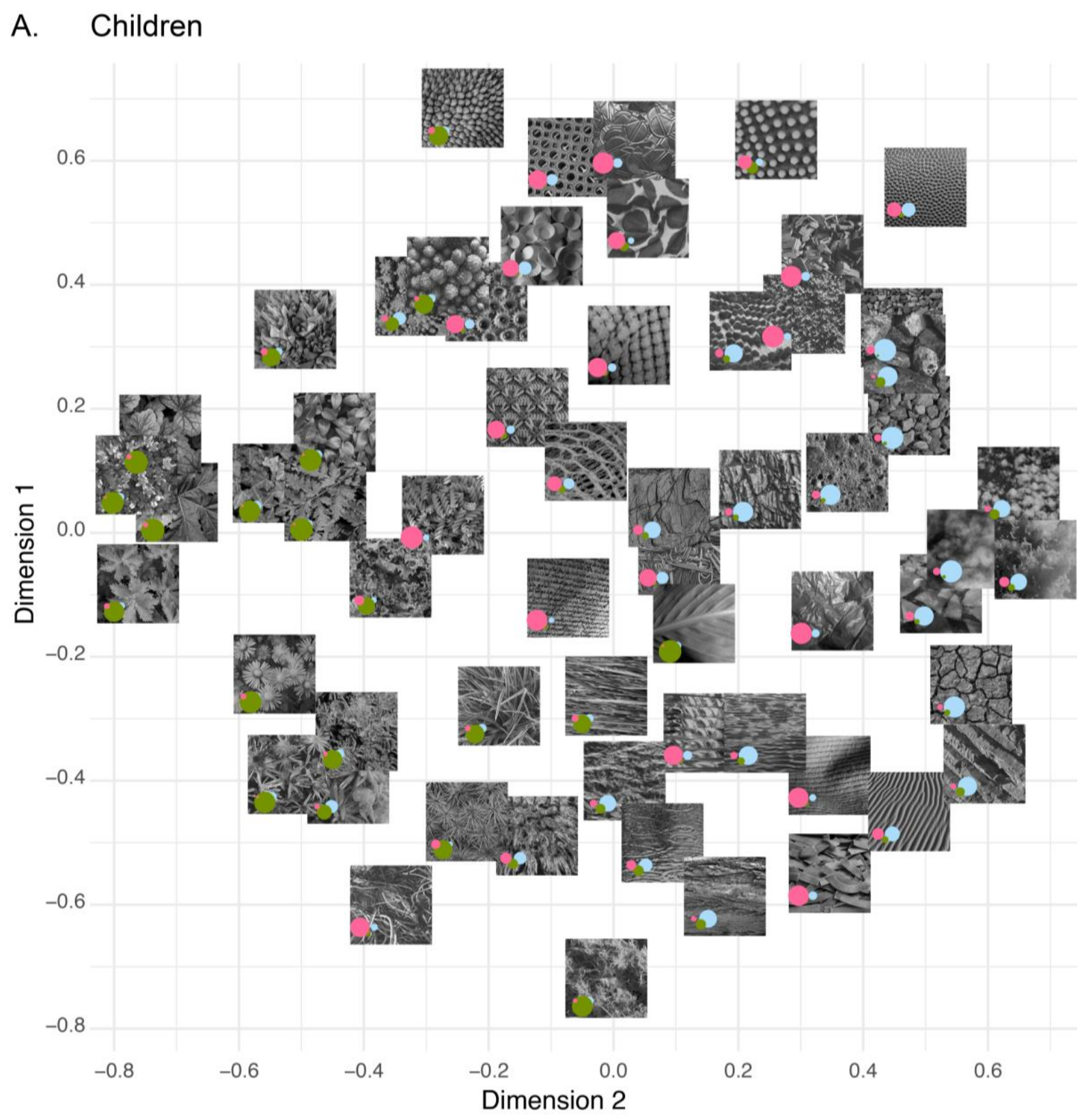


B. Adults

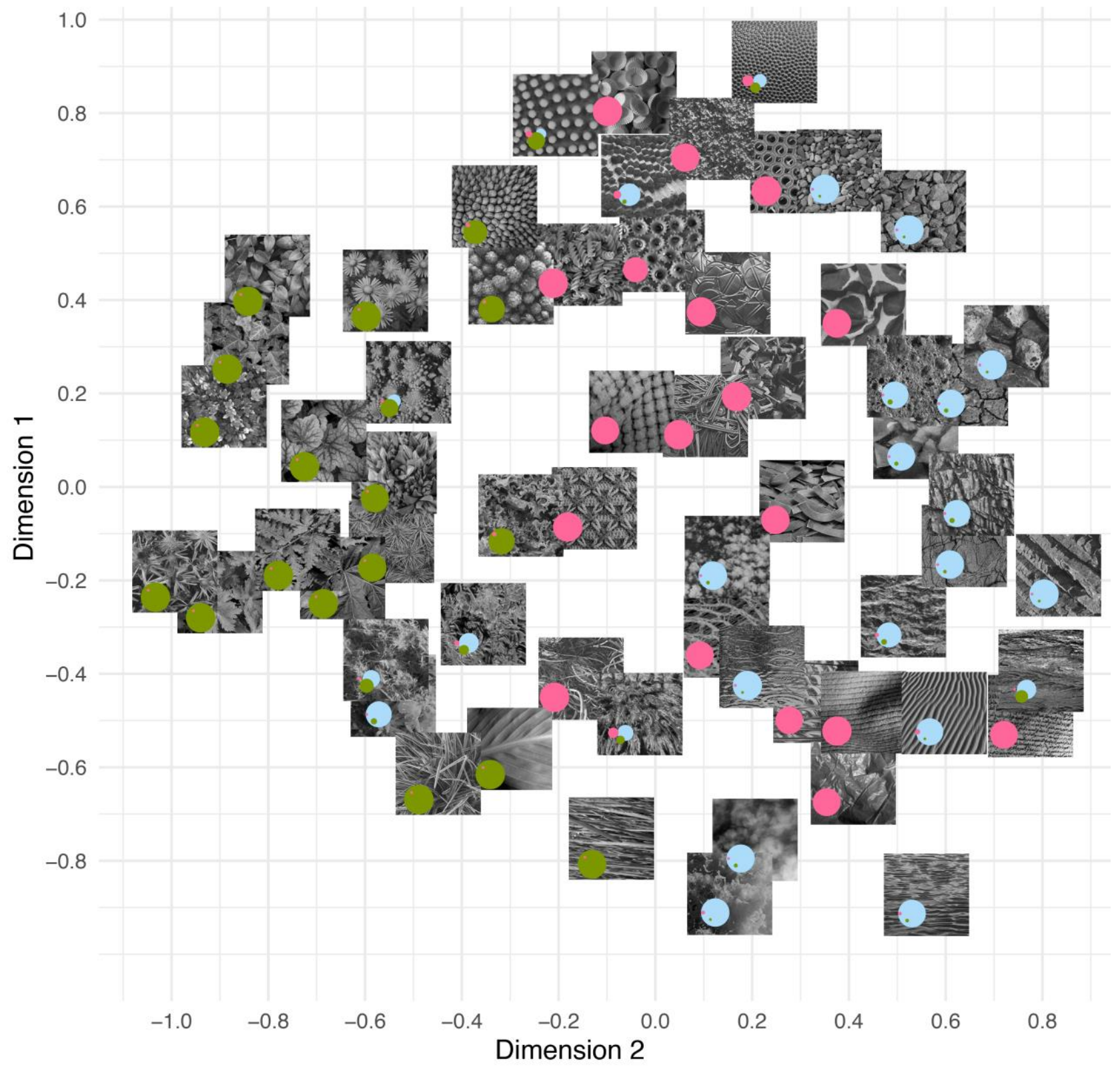

Assigned to: Artifact Natural element Vegetation

Figure 8. Two-dimensional nMDS solutions and the distribution of assigned categories.

Images used in the task are plotted in configurations obtained by nMDS models, for children (A) and adults (B). Stress fit index was .293 for children, and .252 for adults. We applied Procrustes transformation to the models to obtain comparability between the participant groups (congruence coefficient $=.93$ ). The size of the colored discs superimposed on each image represents the proportional assignments of categories to these images by the participant groups (see also Figure 2). 
CHILDREN AND ADULTS, DISTINGUISHING NATURALISTIC IMAGES

Inspection of the nMDS configurations allows inferences about the combinations of image characteristics which might have been affecting similarity judgments. This is of interest because children differ from adults in their ability to differentiate combinations of visual properties (e.g., Smith, 1979). On first sight, nMDS configurations of children and adults (Figure 8) show generally similar arrangements. For example, the bottom right corners include images with elongated, parallel elements, and the top of the configurations include images depicting small distributed particles. Next to this more general overlap, disparities in the characteristics of more direct image neighbors are noticeable. In the children's nMDS, image neighbors frequently overlapped in clearly visual characteristics. For example, the 4image-group at the crossing of dimension $1(\mathrm{D} 1)=.4$, and D2 $=-.3$ to -.1 shows round, erected elements with jagged contours, or the 3-image group at D1 -.4, D2 = -.4 includes radially-configured elongated elements. In contrast, adults also appeared to use non-visual similarities to assemble images. One example is the 2-image-group at D1 $=-.5$ to -.4 , D2 $=-.2$ to 0 , in which two artifacts with irregular structured threads and cables are assembled in spite of their different patterns. Another example is D1 $=.1$ to .3 , D2 $=.5$ to .7, where a 4-imagegroup of natural elements overlaps in some image characteristics (i.e., shape, material, or the subcategory stone surfaces), but none seems to be included in every image. To a lesser degree, these kinds of thematic combinations are also obvious in children, such as the two crochet works at $\mathrm{D} 1=.1$ to $.2, \mathrm{D} 2=-.1$. These examples are intended to provide illustration of how similarity might be perceived in children and adults.

For children and adults, assigned vegetation was a major image characteristic related to similarity decisions (Figure 6 andFigure 7). In the nMDS configurations, we found that children and adults organized the images in accordance with their assigned categories (Figure 8). In particular, images on the left tended to predominantly depict vegetation. Artifacts and natural elements shared the remaining space, with less clearer boundaries. In the HCA, vegetation was the strongest predictor for both participant groups' sorting, especially within the organization of images to larger clusters.

In order to gain a deeper understanding of general factors influencing the participant groups' similarity judgments, we conducted a post-hoc analysis of participants' criteria for determining similarity. We assessed these criteria in adults with a questionnaire asking which 
factors they prioritized in their similarity judgments, and we recorded the children's spontaneous comments about the images during the sorting task (see SI, A.8). According to this assessment, children referred to the structures' appearance in $62 \%$ of their comments during similarity sorting, and in $38 \%$ to their identity. Adults based their similarity judgments to $55 \%$ on appearance, and on $45 \%$ on criteria related to the identity of the structure. This indicates that children and adults attended to visual appearance as well as the depicted entities during the sorting task. For further information about the procedure and results see SI, A.8, and Table A.4.

\section{Discussion}

In the present study, we investigated 4- to 5-year-old children's and adults' categorization of images depicting real-world structures by focusing on the predictive value of visual properties and superordinate category membership on categorization performance. Participants performed a sorting task in which they assembled a set of images into groups according to visual similarity, and a classification task in which another set of images was put into boxes representing the superordinate categories artifacts, natural elements, and vegetation. We then related the sorting decisions of the participant groups to the visual properties and categories of the images.

The results of both tasks show that preschool children readily perceive and interpret complex visual structures by 4 years of age. Within the child participant group, correct classifications increased with the child's age. At the same time, their sorting decisions differed from those of the adults. In particular, children's ability to discriminate the superordinate categories was generally lower than that of adults. Moreover, the predictive value of many visual properties for assigned category membership and judgments of similarity was lower in children than in adults. These general differences might result from stronger inconsistencies in the children's choices of category assignments to the images. The classification task was designed as a three-alternative forced choice paradigm to be appropriate for this age range (see also: Badger \& Shapiro, 2012; Coley et al., 2005; Soja et al., 1991). We intended to elicit inferences about category membership based on perceptual information, in case the images were unfamiliar to the participant. To some degree, children's performance may also be 
affected by inattentiveness or other forms of distraction that typically introduce noise in data from young children. Nevertheless, variations in noise between the assessed visual properties or categories very likely point to differences in children's sensitivity to particular image characteristics. Adults' sorting decisions can thereby serve as a comparison and inform us about the potential predictive power of our property selection for adult-like classification. Consequently, in the following discussion, we are mainly interested in differences between the visual properties' and superordinate categories' predictive power within children relative to adults. Indeed, some of the significant properties did not differ between children and adults, or predicted a category even more strongly in children than in adults, indicating that children also showed systematic sensitivities for image characteristics. We will start with the discussion of visual properties, followed by higher order factors, and then discuss the significance of superordinate category membership.

\section{Visual Properties Predicting Task Performance}

In the classification task, the statistical properties deviation and skew, and the rated properties pictorial depth and symmetry predicted children's assignment to categories, whereas adults' classification was additionally predicted by alpha, curvature, and size. In contrast to the weaker predictive value of most properties for children's classification, low deviation more strongly predicted their assignments of images to vegetation compared to adults. Children's similarity judgments were most strongly predicted by variations in the property depth, followed by skew and regularity, whereas adults' judgments were most strongly predicted by variations in regularity, followed by symmetry and deviation (see SI, A.6, Figure A.3). A comparison of the participant groups' similarity judgments revealed that they did not differ in the high predictive value of depth and skew — nor in the equally weak property alpha (Figure 7).

\section{Does the Experience of Scaling Invariance Increase Children's Sensitivity to Spatial Frequency Distributions?}

Children's sensitivity to the property deviation during classification suggests that a statistical property which is rather abstract and predominantly processed preattentively in adults might be rather essential in young children. High values of deviation indicate the 
absence or dominance of particular spatial frequencies. Artifacts with their even surfaces or repetitive elements frequently possess this characteristic, and high deviation predicted children's and adults' assignments to artifacts. In contrast, the marginal mean of the deviation property in children's assignments to vegetation was significantly lower than in adultsdespite the significant contribution of deviation to adults' vegetation assignments. Moreover, deviation predicted children's similarity perception rather moderately, except at a point in the HCA clustering sequence in which vegetation increased its impact (height 1.2 to 1.5, Figure 6). Low values of deviation meet the quality of scaling invariance. The peculiarity of scaling invariance can be experienced: When moving through a natural environment or approaching an image depicting such a scene, from any viewing distance, the amplitude spectrum of lightto-dark changes (i.e., spatial frequencies) remains approximately the same. Scaling invariance is a typical quality of natural scenes (Ruderman, 1997). Thus, visual structures that are scaling invariant can be seen as qualitatively different from structures that are not scaling invariant (i.e., high in the property deviation), because movement uncovers variations in this property and may support sensitivity to its variability. A particular sensitivity to deviation was even found in an eye-tracking search task with 8-month-olds that was using the same images (Schlegelmilch \& Wertz, 2022), where stronger differences in deviation between the target image patch and the background image increased infant's detection success.

The current results suggest that children over-generalized vegetation to unfamiliar images on the basis of visual regularities that were gathered from their environment (i.e., low deviation). A similar phenomenon was described by Balas (2017) in a material categorization task with 5- to 7-year-olds, suggesting that available but incomplete visual information had led to quickly-adopted representations which then led to incorrect responses.

But why were children sensitive to variations in deviation, but not to variations in alpha, that refers to the steepness of spatial frequencies distributions? Alpha significantly predicted artifacts and natural elements in adults' classification and in the image set. The current low predictive value of alpha for children's category assignments replicates earlier findings of Ellemberg and colleagues $(1999,2012)$ who argued that preschool children's sensitivity to high spatial frequencies, and to low spatial frequencies with fine contrasts is still immature. Processing advantages for typical alpha ranges (e.g., in natural scenes) which are common in adults are therefore not available to children below 10 years of age. This 
CHILDREN AND ADULTS, DISTINGUISHING NATURALISTIC IMAGES

immature sensitivity to low and high spatial frequencies was even more evident for naturalistic images than for graphic stimuli (Ellemberg et al., 2009). However, the spatial frequency scales that were less available to children in Ellemberg and colleagues $(1999,2012)$ are similarly assessed in deviation and in alpha, so that further reasons must underlie the current differences in children's sensitivity to these properties.

Possibly, sensitivity to variations in alpha relies on more complex visual processing steps than deviation. Variations in alpha are based on relative differences in the proportions of spatial frequency magnitudes: if the spatial frequency distribution falls steeper, alpha refers to greater energy in low spatial frequencies than high spatial frequencies (Figure 1). In contrast, variations between low and high deviation indicate the absence or dominance of particular spatial frequencies. Thus, alpha relies on more complex neural computations than deviation. Additionally, the experiential nature of deviation's scaling invariance could lead to an earlier sensitivity to deviation - variations in alpha cannot be easily resolved in explorative actions, but deviation's scaling invariance can be experienced during movements. Moreover, one might conjecture that due to this quality, it is not necessary to perceive the full range of spatial frequencies included in low values of deviation, because the overall impression of the available visual information remains the same. Children might benefit from such visual characteristics, in particular if they refer to significant category distinctions.

Rated pictorial depth was an important predictor for children's sorting decisions. Depth is of ecological significance because it is closely entangled with scene segmentation and sensorimotor coordination (Gibson, 2000; Kellman \& Shipley, 1991). Sensitivity to monocular depth cues such as contour junctions, shading, or texture gradients in graphic stimuli is already present in infants around 6 months of age (Kavšek et al., 2012). Such early onset of sensitivity to pictorial depth indicates the relevance of this property. In real-world situations, three-dimensional depth of spatial arrangements can be solved by children's explorative movements (Bertenthal, 1996; Campos et al., 2000) and thereby provide signals of pictorial depth (Nardini et al., 2010).

However, previous research has reported immature analyses of pictorial depth until middle childhood. Freud \& Behrmann (2017) compared sensitivity of children and adults to graphics that either obeyed or violated possible 3D structure. In this task, children younger than 6.5 years were not able to compute the fine-grained depth descriptions of the images. It is 
possible that the depth cues in our naturalistic images were easier to perceive for children because, in contrast to Freud and Behrmann (2017), no detailed analysis of contour junctions was necessary. Additionally, depth in the current images is strongly supported by shading information. The availability of shading information can have made the analysis of finegrained and distributed pictorial depth cues (e.g., contour junctions) unnecessary, which is more demanding for young children than differences in luminance (Sireteanu et al., 2005; Taylor et al., 2014).

These examples with deviation and pictorial depth confirm that visual properties of the environment that children themselves can trigger or manipulate play a particular role during the development of categorization (see also: Ballard et al., 1997; Bertenthal, 1996; Schmuckler, 1993). Children may primarily learn such properties (Köster et al., 2020; Oudeyer \& Smith, 2016), and they may be beneficial to solve visual tasks during development if more complex visual properties are not available.

\section{Did Children Predominantly Attend to More Global Image Properties?}

Skew strongly predicted children's sorting decisions. Similar to depth, variations in skew were possibly perceived instantaneously by children. Skew is necessarily perceived more globally because it refers to the differences in the overall grey levels an image is composed of - independent of any shape details or organizational demands. In greyscale images, skew gives an impression of the structures original brightness, which is mainly based on color pigmentation and lighting conditions (Kim \& Anderson, 2010). Contrasts in brightness and color are closely intermingled in many visual processing stages (Gegenfurtner \& Kiper, 2003), and both properties contribute significantly to the identification and differentiation of structures within the environment (Gegenfurtner \& Rieger, 2000). If color is not available, the distribution of an image's brightness levels that carry information about pigmentation possibly support categorization. Thus, children's adult-like sensitivity to skew during similarity judgments and classification might rely on its presumably low processing effort and its applicability as color alternate.

In contrast to depth and skew, the properties symmetry and curvature, which are both shape related properties, strongly predicted adults' similarity judgments, but were less significant for children's judgments. Moreover, in the classification task, symmetry also 
differed between children and adults for each of the three assigned categories. There is evidence that the development of symmetry perception is still ongoing during preschool age (Bornstein \& Stiles-Davis, 1984), whereas curvature cues were already found to attract attention during infancy (Kellman \& Arterberry, 2007). The most likely explanation for the lower impact of these shape properties could lie in the cognitive demand on young children raised by a necessary integration of detailed visual cues into larger shapes (Fornari et al., 2014). In complex naturalistic structures, the perception of shape is part of a hierarchical organizational process and relies on several perceptual mechanisms: smaller elements can be grouped, compared, segregated, or perceived in their configural relations, leading to more global elements with which the operations can be repeated (Kimchi, 2015; Wagemans et al., 2012). The instantaneous perception of mid-level shape elements in naturalistic structures may be difficult for a preschooler's maturing visual system. Immature vernier acuity and less efficient read-out of visual information between low- and higher-order visual processing stages affects the visuo-spatial extraction of shape elements beyond childhood (e.g., Dekker et al., 2019; Skoczenski \& Norcia, 2002).

In order to receive additional insight into the effect of maturing visual abilities, we conducted a post-hoc analysis of the children's classification data which included the continuous variables age, visual property, and the interaction term Age $\times$ Visual Property. With this, we aimed to investigate age-related changes in the predictive value of visual properties which rely on shape and detail. And indeed, as shown in the SI, A.11, Figure A.8, properties that rely on hierarchical processing (symmetry, size, curvature, regularity), or on detail (CooCor) predicted the older children's classification in some categories in a more differentiated way. In contrast, depth and skew did not differ between younger and older children's classification, indicating that 4-year-olds similarly integrated these predictors in their category assignments (see SI, A.11 for the full analysis).

Regularity predicted children's similarity judgments as much as skew. Regularity provides an overall impression of the structure of an image. Thus, children must have been able to distinguish between irregular and regular arrangements during similarity sorting. Yet, regularity was more differentiated in older than younger children in the classification task (SI, A.11) - the extraction and higher order integration of mid-level elements might still be more effortful in younger compared to older preschoolers. 
When discussing significant age-related increases in children's sensitivity to visual properties, it is important to consider alternative reasons. For example, it is intriguing that age-related changes in children's sensitivities to properties seemed to depend on a particular category: None of the properties in children's assignments to vegetation changed with age. Possibly, this indicates age differences in the clarity of superordinate-category representations. We will discuss this in more detail later.

Preliminarily, we can summarize that particular visual properties that are defined by rather global or holistic characteristics predicted children's categorization more strongly than properties that referred to mid-level shape elements or that relied on a more complex analysis of distributed information.

\section{Perceptual Integration of Naturalistic Structure Components}

The preceding results suggest that hierarchical organizational processes affect children's perception of naturalistic structures. However, the generally stronger predictive value of global image properties for children's card sorting is in contrast to the hypothesis that processing advantages for local detail and local superiority still extends over middle childhood even for naturalistic images (e.g., Balas, Auen, Thrash, et al., 2020; Scherf et al., 2009). Why, then, did detail-related properties not predict children's current sorting decisions more reliably?

Possibly, children's attentional focus was also affected by factors exceeding the individual visual structures depicted on the cards. For example, adults show evidence for global precedence or coarse-to-fine processing hierarchies, but are also flexibly integrating different hierarchical levels depending on context, attentional deployment, or the aspect under investigation (Flevaris \& Robertson, 2016; Hegdé, 2008). Similar to adults, children's preference to process global or local graphic features were also affected by a particular task, by the density and number of the elements, or their being object or non-object (reviewed in: Guy et al., 2013; Kimchi, 2015; for attention to detailed and whole figural contours see also: Lange-Küttner et al., 2002; M. Barrett \& Eames, 1996). And indeed, a comparison of the two tasks suggests that a flexible integration of visual properties occurred to some extent in both participant groups. 
Children might have adapted visual strategies to solve the respective tasks. For example, informational density was lower during classification with one image viewed at a time, facilitating an analytic investigation of shape details to solve classification (e.g., Deng \& Sloutsky, 2016; Rosch et al., 1976). In contrast, many images were presented simultaneously during similarity sorting, leading to a more general view on the images (e.g., configural or shading properties). Correspondingly, in children, (a) sensitivity to the holistic property regularity increased during similarity grouping compared to classification, (b) distinctions of mid-level shape characteristics between non-symmetrical versus symmetrical forms were present during classification, but reduced during similarity grouping, and (c) sensitivity to low versus high deviation was more dominant during classification than during similarity grouping. In contrast, skew or rated depth predicted categorization more generally in both tasks. Perceptual organization of high density and wider distributed visual information could have posed greater effort on children than on adults (Balas, Auen, Thrash, et al., 2020; Hadad et al., 2010; Hadad \& Kimchi, 2006). The greater effort may depend on the still increasing horizontal intra- and interhemispheric connectivity, as well as increasing feedback connectivity from extrastriate visual areas to V1 during childhood. These changes are thought to be involved in the detection, grouping and spatial integration of distributed visual elements (Fornari et al., 2014; Knyazeva, 2013; Kovács, 2000; van den Boomen et al., 2014).

In accordance with the above, we reason that preschool children flexibly focused on different levels of the local-global hierarchy, possibly affected by the density and spread of visual information, and by the tasks' demands. Children's flexible integration of available visual information could reflect their visual strategies in naturalistic environments, where figure-ground relationships are not clearly defined. Hierarchical levels in every-day surroundings are fluent, so that the size of the attentional spotlight can depend on the observer's choice of informational resolution or their processing capacity.

\section{Assigned Category Membership Predicted Similarity Judgments in Both Participant Groups}

The superordinate categories that were assigned to the images by the participant groups during classification predicted their similarity decisions. Young children as well as adults seem to have processed images in a semantic fashion, while simultaneously the 
available combinations of images triggered category formation based on their visual properties (for similar suggestions see: Hoehl, 2016; Mandler, 2000). This would indicate an equivalent inclusion of visual properties and semantic categories in similarity perception (see also SI, A.8 and Table A.5).

Of the three categories, assigned vegetation was the strongest predictor for similarity in children and adults. Vegetation had a very high impact in the HCAs at organizations of the images to only few clusters, indicating a domain-like differentiation (Figure 6). Such a differentiation is also reflected in the nMDS solutions, where natural elements and artifacts do not seem to be separated as clearly from each other as from vegetation (Figure 8). Despite children's generally lower classification performance, both children and adults showed lower discriminability for natural elements than for vegetation and artifacts. Children also showed a higher relative discriminability for vegetation than adults did. In addition, the fact that children showed similar levels of discriminability for artifacts and vegetation suggests that preschool children possess a superordinate category representation for plants (see e.g., Gelman, 2004) similar to their category representation for artifacts (e.g., Casler \& Kelemen, 2005), complementing previous findings on preschoolers' rich conceptual representation of plants (e.g., Hickling \& Gelman, 1995; Nguyen \& Gelman, 2002). Our findings reveal that beyond conceptual characteristics, particular visual properties - namely greater depth and low deviation-predicted preschoolers' assignments to vegetation when compared to artifacts and natural elements.

Intriguingly, there were no age-related differences in the properties of images that children assigned to vegetation, whereas properties in images assigned to artifacts and also to natural elements were more age-dependent (SI, A.11, Figure A.8). Increasing differentiation of properties predicting artifact assignments may result from the importance of experiences with the specific purpose or usage of objects, that determine representations of the artifact category (Carrara \& Mingardo, 2013; Rakison \& Yermolayeva, 2010b). It may also underlie the ability to recognize objects and tools from unusual perspectives, that is found to still develop during school age (Bova et al., 2007; Dekker et al., 2011) and applies to some of the structure elements of our images (as ensemble or close-up). In contrast, assignments to vegetation were predicted in our set of properties with more age-related stability. This is surprising in particular for the property deviation, because its predictive value for children's 
category assignments to all three categories differed from adults. Nevertheless, the phenomenon of scaling invariance (i.e., low deviation) seems to have been attributed to vegetation similarly in 4- and 5-year-olds.

To ensure that an increase in assembled vegetation images did not result from more similar images within vegetation than within the other categories, we analyzed whether the variance of the image properties differed between the categories. This analysis shows that statistically, there were no differences in the variance of the visual properties between the categories, SI, A.12.

Research with infants provides evidence that sensitivity to plants starts to develop very early in life. Infants treat plants differently than artifacts and other natural kinds (Elsner \& Wertz, 2019; Mandler \& McDonough, 1998; Wertz \& Wynn, 2014; Włodarczyk et al., 2018), suggesting that they are able to visually distinguish visual properties of the plants from those of the other types of entities which were used as stimuli in the studies. That the categorization of what is assumed to be vegetation in the current study is only marginally influenced by the age of the child may indicate that from early development on, the categorization of objects or visual structures as vegetation is of particular significance (see e.g., Wertz, 2019).

\section{Limitations}

The current results were obtained by comparing assigned images of one category to the two remaining categories. Thus, the visual properties that significantly predicted classification decisions do not provide absolute descriptors of properties relevant for the classification of artifacts, natural elements, and vegetation. However, by comparing significant superordinate categories which differ in several conceptual and functional aspects, we intended to extract visual properties which add to an understanding of processes and sensitivities underlying visual categorization in naturalistic environments.

The visual properties we examine here were intended to represent a broad sample relevant to the research, but they are not exhaustive. In addition to further variants of statistical or rated properties, classification decisions may have also been affected by characteristics that are more closely entangled with concepts about shape (e.g., "showing a main part" was a characteristic of animals in Schmidt et al., 2017). For example, Figure 2 shows that of the true natural elements that children assigned to the vegetation category, 
crystals were the most-represented sub-category. Crystals are formed by crystal growth, which is visually reflected in their shape and resembles, to some degree, the growth of plants. Moreover, crystals might not be as familiar to children as other natural elements (e.g., pebbles or clouds). Such concept-related characteristics were not assessed in the current study, but might provide additional interesting insight into categorization development in future research. Nevertheless, we expect that our results will contribute to the understanding of sensitivities to particular visual properties during the development of visual categorization, and to the interrelation between these sensitivities and the particular significance of a category for a respective age-group.

\section{Conclusion}

In the current study we explored visual properties that predict preschool children's and adults' distinctions of complex naturalistic stimuli. Our findings show that some visual properties similarly predicted categorization decisions in both participant groups, but there were also systematic differences. Visual properties that vary due to explorative movements (i.e., depth, scaling invariance), or properties that can be perceived holistically (skew, depth relying on shading information, regularity) predicted children's categorization decisions more strongly, whereas properties that relied more on higher order visual computations predicted children's decisions less strongly (e.g., variations in the shape of structure elements like their symmetry or curvature, or comparisons of the distribution of spatial frequency magnitudes that define the property alpha). Compared to adults, children's categorization seems to be based less on these higher-order computations, because they require increased visual processing effort.

In the current study, properties at different hierarchical processing levels nevertheless predicted children's categorization depending on the categorization task, and the number of images presented. This suggests that preschool children have detected-and flexibly integrated - task-relevant visual information in accordance with their visual processing capacities. This finding is important, because in complex naturalistic stimuli, visual hierarchies are more fluent and ambiguous than in the graphically structured stimuli of common global-local paradigms (Kimchi, 2015), so that the current findings add to the sparse 
developmental literature investigating local-global processing with naturalistic stimuli (e.g., Balas, Auen, Saville, et al., 2020; Balas, Auen, Thrash, et al., 2020).

Of the categories assigned to images by children and adults, vegetation was the strongest predictor of similarity in both participant groups. Moreover, children's assignments to vegetation were also predicted by our set of properties with more age-related stability, whereas the differentiation of properties in images assigned to artifacts increased with children's age. This suggests a general sensitivity in 4- to 5-year-olds to the vegetation category, while the gathering of visual characteristics of the artifact category is still ongoing.

The current project shows that research on visual categorization development with complex naturalistic image stimuli expands research using bounded or graphic stimuli. It also provides examples of children's integration of visual properties during categorization in different contexts and tasks. The explorative nature of the current investigation will benefit from follow-up studies investigating categorization development with ecologically significant categories presented as naturalistic images in additional age groups (see also: Schlegelmilch \& Wertz, 2022).

The data underlying the statistical analysis of this study is accessible under the link https://osf.io/8xy5n/?view_only=6ddced286c31456fae7d20dd86e072e6.

\section{Acknowledgements}

We thank B. Lewke, J. Eichelsdörfer, T. Pokojski, C. Kaiser, D. Tatlow-Devally, M. Wiedenhöft, D. Erdt, H. Nassimi and the members of the Max Planck Research Group Naturalistic Social Cognition for their assistance. We thank Moritz Daum and the members of the Department of Developmental Psychology: Infancy and Childhood at the University of Zurich, Damian Läge, Karl Gegenfurtner and members of the Department of Experimental Psychology at Justus-Liebig-University Giessen, for the supporting discussions. We especially thank our participants and their parents.

\section{Conflict of Interest}

We have no known conflict of interest to disclose. 


\section{References}

Açık, A., Onat, S., Schumann, F., Einhäuser, W., \& König, P. (2009). Effects of luminance contrast and its modifications on fixation behavior during free viewing of images from different categories. Vision Research, 49(12), 1541-1553. https://doi.org/10.1016/j.visres.2009.03.011

Almoqbel, F. M., Irving, E. L., \& Leat, S. J. (2017). Visual acuity and contrast sensitivity development in children: Sweep visually evoked potential and psychophysics. Optometry and Vision Science, 94(8), 830-837.

Amso, D., Haas, S., \& Markant, J. (2014). An eye tracking investigation of developmental change in bottom-up attention orienting to faces in cluttered natural scenes. PloS One, 9(1), e85701.

Aslin, R. N., \& Smith, L. B. (1988). Perceptual Development. Annual Review of Psychology, 39(1), 435-473.

Atkinson, J., \& Braddick, O. (2013). Visual Development. The Oxford Handbook of Developmental Psychology, Vol. 1. https://doi.org/10.1093/oxfordhb/9780199958450.013.0010

Backscheider, A. G., Shatz, M., \& Gelman, S. A. (1993). Preschoolers' ability to distinguish living kinds as a function of regrowth. Child Development, 64(4), 1242-1257.

Badger, J. R., \& Shapiro, L. R. (2012). Evidence of a transition from perceptual to category induction in 3- to 9-year-old children. Journal of Experimental Child Psychology, 113(1), 131-146. https://doi.org/10.1016/j.jecp.2012.03.004

Balas, B. (2017). Children's use of visual summary statistics for material categorization. Journal of Vision, 17(12), 22-22. https://doi.org/10.1167/17.12.22

Balas, B. (2021). Texture Perception. In Oxford Research Encyclopedia of Psychology. Oxford University Press. https://doi.org/10.1093/acrefore/9780190236557.013.884

Balas, B., Auen, A., Saville, A., Schmidt, J., \& Harel, A. (2020). Children are sensitive to mutual information in intermediate-complexity face and non-face features. Journal of Vision, 20(5), 6-6. https://doi.org/10.1167/jov.20.5.6

Balas, B., Auen, A., Thrash, J., \& Lammers, S. (2020). Children's use of local and global visual features for material perception. Journal of Vision, 20(2), 10. https://doi.org/10.1167/jov.20.2.10

Balas, B., \& Saville, A. (2021). Neural sensitivity to natural image statistics changes during middle childhood. Developmental Psychobiology, 63(5), 1061-1070. https://doi.org/10.1002/dev.22062

Balas, B., Saville, A., \& Schmidt, J. (2018). Neural sensitivity to natural texture statistics in infancy. Developmental Psychobiology, 60(7), 765-774.

Balas, B., \& Woods, R. (2014). Infant Preference for Natural Texture Statistics is Modulated by Contrast Polarity. Infancy, 19(3), 262-280. https://doi.org/10.1111/infa.12050

Ballard, D. H., Hayhoe, M. M., Pook, P. K., \& Rao, R. P. (1997). Deictic codes for the embodiment of cognition. Behavioral and Brain Sciences, 20(4), 723-742.

Barrett, H. C. (2014). The shape of thought: How mental adaptations evolve. Oxford University Press.

Barrett, M., \& Eames, K. (1996). Sequential developments in children's human figure drawing. British 
CHILDREN AND ADULTS, DISTINGUISHING NATURALISTIC IMAGES

Journal of Developmental Psychology, 14(2), 219-236.

Bates, D., Mächler, M., Bolker, B., \& Walker, S. (2015). Fitting Linear Mixed-Effects Models Using lme4. Journal of Statistical Software, 67(i01).

Baumgartner, E., \& Gegenfurtner, K. R. (2016). Image Statistics and the Representation of Material Properties in the Visual Cortex. Frontiers in Psychology, 7. https://doi.org/10.3389/fpsyg.2016.01185

Baumgartner, E., Wiebel, C. B., \& Gegenfurtner, K. R. (2013). Visual and Haptic Representations of Material Properties. Multisensory Research, 26(5), 429-455. https://doi.org/10.1163/2213480800002429

Behrensmeyer, A. K. (1982). The geological context of human evolution. Annual Review of Earth and Planetary Sciences, 10, 39.

Benjamini, Y., \& Hochberg, Y. (1995). Controlling the false discovery rate: A practical and powerful approach to multiple testing. Journal of the Royal Statistical Society: Series B (Methodological), 57(1), 289-300.

Bertenthal, B. I. (1996). Origins and Early Development of Perception, Action, and Representation. Annual Review of Psychology, 47(1), 431-459. https://doi.org/10.1146/annurev.psych.47.1.431

Bhatt, R. S., \& Quinn, P. C. (2011). How Does Learning Impact Development in Infancy? The Case of Perceptual Organization: PERCEPTUAL LEARNING IN INFANCY. Infancy, 16(1), 2-38. https://doi.org/10.1111/j.1532-7078.2010.00048.x

Bondarko, V. M., \& Semenov, L. A. (2012). Visual acuity and hyperacuity in 11-to 17-year-old secondary school students. Human Physiology, 38(3), 271-275.

Bornstein, M. H., \& Stiles-Davis, J. (1984). Discrimination and memory for symmetry in young children. Developmental Psychology, 20(4), 637.

Bova, S. M., Fazzi, E., Giovenzana, A., Montomoli, C., Signorini, S. G., Zoppello, M., \& Lanzi, G. (2007). The development of visual object recognition in school-age children. Developmental Neuropsychology, 31(1), 79-102.

Braddick, O., \& Atkinson, J. (2011). Development of human visual function. Vision Research, 51(13), 1588-1609. https://doi.org/10.1016/j.visres.2011.02.018

Brant, L. A. (1987). A Case for Geology. Iowa Science Teachers Journal, 24(2), 2-5.

Burton, G. J., \& Moorhead, I. R. (1987). Color and spatial structure in natural scenes. Applied Optics, 26(1), 157-170.

Campos, J. J., Anderson, D. I., Barbu-Roth, M. A., Hubbard, E. M., Hertenstein, M. J., \& Witherington, D. (2000). Travel broadens the mind. Infancy, 1(2), 149-219.

Carey, S. (1988). Conceptual differences between children and adults. Mind \& Language, 3(3), 167181.

Carrara, M., \& Mingardo, D. (2013). Artifact Categorization. Trends and Problems. Review of Philosophy and Psychology, 4(3), 351-373. https://doi.org/10.1007/s13164-013-0151-6

Casler, K., \& Kelemen, D. (2005). Young children's rapid learning about artifacts. Developmental 
Science, 8(6), 472-480.

Clausi, D. A. (2002). An analysis of co-occurrence texture statistics as a function of grey level quantization. Canadian Journal of Remote Sensing, 28(1), 45-62.

Coley, J. D., Seaton, C. E., Vitkin, A. Z., \& Yopchick, J. E. (2005). Effects of experience on relational inferences in children: The case of folk biology. Proceedings of the Annual Meeting of the Cognitive Science Society, 27(27).

Costa, A. F., Humpire-Mamani, G., \& Traina, A. J. M. (2012). An efficient algorithm for fractal analysis of textures. Graphics, Patterns and Images (SIBGRAPI), 2012 25th SIBGRAPI Conference On, 39-46.

Daw, N. W. (2014). Visual Development. Springer US. https://doi.org/10.1007/978-1-4614-9059-3

De Leeuw, J., \& Mair, P. (2011). Multidimensional scaling using majorization: SMACOF in R.

Dekker, T. M., Mareschal, D., Sereno, M. I., \& Johnson, M. H. (2011). Dorsal and ventral stream activation and object recognition performance in school-age children. NeuroImage, 57(3), 659670 .

Dekker, T. M., Schwarzkopf, D. S., de Haas, B., Nardini, M., \& Sereno, M. I. (2019). Population receptive field tuning properties of visual cortex during childhood. Developmental Cognitive Neuroscience, 37, 100614. https://doi.org/10.1016/j.den.2019.01.001

Deng, W. (Sophia), \& Sloutsky, V. M. (2016). Selective attention, diffused attention, and the development of categorization. Cognitive Psychology, 91, 24-62.

https://doi.org/10.1016/j.cogpsych.2016.09.002

Elder, J. H., \& Goldberg, R. M. (2002). Ecological statistics of Gestalt laws for the perceptual organization of contours. Journal of Vision, 2(4), 5-5.

Ellemberg, D., Hansen, B. C., \& Johnson, A. (2012). The developing visual system is not optimally sensitive to the spatial statistics of natural images. Vision Research, 67, 1-7. https://doi.org/10.1016/j.visres.2012.06.018

Ellemberg, D., Johnson, A., \& Hansen, B. (2009). The development of contrast sensitivity for gratings and natural images: Revisiting the golden standard. Journal of Vision, 9(8), 984-984. https://doi.org/10.1167/9.8.984

Ellemberg, D., Lewis, T. L., Liu, C. H., \& Maurer, D. (1999). Development of spatial and temporal vision during childhood. Vision Research, 39(14), 2325-2333.

Elsner, C., \& Wertz, A. E. (2019). The seeds of social learning: Infants exhibit more social looking for plants than other object types. Cognition, 183, 244-255.

https://doi.org/10.1016/j.cognition.2018.09.016

Emberson, L. L., \& Rubinstein, D. Y. (2016). Statistical learning is constrained to less abstract patterns in complex sensory input (but not the least). Cognition, 153, 63-78.

Fantz, R. L., \& Nevis, S. (1967). Pattern preferences and perceptual-cognitive development in early infancy. Merrill-Palmer Quarterly of Behavior and Development, 13(1), 77-108.

Field, D. J. (1987). Relations between the statistics of natural images and the response properties of cortical cells. Journal of the Optical Society of America A, 4(12), 2379-2394. 
https://doi.org/10.1364/JOSAA.4.002379

Fisher, A. V., Godwin, K. E., Matlen, B. J., \& Unger, L. (2015). Development of Category-Based Induction and Semantic Knowledge. Child Development, 86(1), 48-62. https://doi.org/10.1111/cdev.12277

Fleming, R. W. (2017). Material Perception. Annual Review of Vision Science, 3(1), 365-388. https://doi.org/10.1146/annurev-vision-102016-061429

Fleming, R. W., Wiebel, C., \& Gegenfurtner, K. (2013). Perceptual qualities and material classes. Journal of Vision, 13(8), 9-9.

Flevaris, A. V., \& Robertson, L. C. (2016). Spatial frequency selection and integration of global and local information in visual processing: A selective review and tribute to Shlomo Bentin. Neuropsychologia, 83, 192-200. https://doi.org/10.1016/j.neuropsychologia.2015.10.024

Fornari, E., Rytsar, R., \& Knyazeva, M. G. (2014). Development of spatial integration depends on topdown and interhemispheric connections that can be perturbed in migraine: A DCM analysis. Neurological Sciences, 35(1), 215-224. https://doi.org/10.1007/s10072-014-1777-6

Fox, J., \& Weisberg, S. (2019). Using car Functions in Other Functions.

Frank, M. C., Vul, E., \& Saxe, R. (2012). Measuring the Development of Social Attention Using FreeViewing. Infancy, 17(4), 355-375. https://doi.org/10.1111/j.1532-7078.2011.00086.x

Freud, E., \& Behrmann, M. (2017). The life-span trajectory of visual perception of 3D objects. Scientific Reports, 7(1), 1-11.

Friesen, M. C., Shortreed, S. M., Wheeler, D. C., Burstyn, I., Vermeulen, R., Pronk, A., Colt, J. S., Baris, D., Karagas, M. R., \& Schwenn, M. (2015). Using hierarchical cluster models to systematically identify groups of jobs with similar occupational questionnaire response patterns to assist rule-based expert exposure assessment in population-based studies. Annals Of Occupational Hygiene, 59(4), 455-466.

Gegenfurtner, K. R., \& Kiper, D. C. (2003). Color vision. Annual Review of Neuroscience, 26(1), 181-206.

Gegenfurtner, K. R., \& Rieger, J. (2000). Sensory and cognitive contributions of color to the recognition of natural scenes. Current Biology, 10(13), 805-808. https://doi.org/10.1016/S09609822(00)00563-7

Geisler, W. S. (2008). Visual perception and the statistical properties of natural scenes. Annu. Rev. Psychol., 59, 167-192.

Geisler, W. S., Perry, J. S., Super, B. J., \& Gallogly, D. P. (2001). Edge co-occurrence in natural images predicts contour grouping performance. Vision Research, 41(6), 711-724.

Gelman, S. A. (1988). The development of induction within natural kind and artifact categories. Cognitive Psychology, 20(1), 65-95. https://doi.org/10.1016/0010-0285(88)90025-4

Gelman, S. A., \& Markman, E. M. (1987). Young children's inductions from natural kinds: The role of categories and appearances. Child Development, 1532-1541.

Gibson, E. J. (2000). Perceptual learning in development: Some basic concepts. Ecological Psychology, 12(4), 295-302. 
Goldstone, R. L. (1998). Perceptual learning. Annual Review of Psychology, 49(1), 585-612.

Goldstone, R. L. (2003). Learning to perceive while perceiving to learn. In Perceptual organization in vision (pp. 245-290). Psychology Press.

Gonzalez, R. C., \& Woods, R. E. (2018). Digital image processing. Pearson.

Graham, D., Schwarz, B., Chatterjee, A., \& Leder, H. (2016). Preference for luminance histogram regularities in natural scenes. Vision Research, 120, 11-21.

https://doi.org/10.1016/j.visres.2015.03.018

Groll, A., \& Tutz, G. (2014). Variable selection for generalized linear mixed models by L 1-penalized estimation. Statistics and Computing, 24(2), 137-154.

Guy, M. W., Reynolds, G. D., \& Zhang, D. (2013). Visual attention to global and local stimulus properties in 6-month-old infants: Individual differences and event-related potentials. Child Development, 84(4), 1392-1406.

Hadad, B., \& Kimchi, R. (2006). Developmental trends in utilizing perceptual closure for grouping of shape: Effects of spatial proximity and collinearity. Perception \& Psychophysics, 68(8), 12641273.

Hadad, B., Maurer, D., \& Lewis, T. L. (2010). The effects of spatial proximity and collinearity on contour integration in adults and children. Vision Research, 50(8), 772-778.

https://doi.org/10.1016/j.visres.2010.01.021

Hair, J. F., Black, W. C., Babin, B. J., Anderson, R. E., \& Tatham, R. L. (1998). Multivariate data analysis (Vol. 5). Prentice hall Upper Saddle River, NJ.

Hansen, B. C., \& Hess, R. F. (2006). Discrimination of amplitude spectrum slope in the fovea and parafovea and the local amplitude distributions of natural scene imagery. Journal of Vision, 6(7), 3. https://doi.org/10.1167/6.7.3

Haralick, R. M., Shanmugam, K., \& Dinstein, I. H. (1973). Textural features for image classification. IEEE Transactions on Systems, Man, and Cybernetics, 6, 610-621.

Hardy, K. (2018). Plant use in the Lower and Middle Palaeolithic: Food, medicine and raw materials. Quaternary Science Reviews, 191, 393-405.

Hardy, K., \& Martens, L. K. (2016). Wild harvest: Plants in the hominin and pre-agrarian human worlds. Oxbow books.

Hartig, F. (2020). DHARMa: Residual Diagnostics for Hierarchical (Multi-Level/ Mixed) Regression Models (0.2.7) [R]. https://CRAN.R-project.org/package=DHARMa

Heaps, C., \& Handel, S. (1999). Similarity and features of natural textures. Journal of Experimental Psychology: Human Perception and Performance, 25(2), 299-320. https://doi.org/10.1037/00961523.25.2.299

Hegdé, J. (2008). Time course of visual perception: Coarse-to-fine processing and beyond. Progress in Neurobiology, 84(4), 405-439.

Hespos, S. J., Ferry, A. L., Anderson, E. M., Hollenbeck, E. N., \& Rips, L. J. (2016). Five-month-old infants have general knowledge of how nonsolid substances behave and interact. Psychological Science, 27(2), 244-256. 
Hickling, A. K., \& Gelman, S. A. (1995). How does your garden grow? Early conceptualization of seeds and their place in the plant growth cycle. Child Development, 66(3), 856-876.

Hiramatsu, C., Goda, N., \& Komatsu, H. (2011). Transformation from image-based to perceptual representation of materials along the human ventral visual pathway. NeuroImage, 57(2), 482-494. https://doi.org/10.1016/j.neuroimage.2011.04.056

Hoffman, D. D., \& Singh, M. (2012). Computational Evolutionary Perception. Perception, 41(9), 1073-1091. https://doi.org/10.1068/p7275

Hurley, K. B., Kovack-Lesh, K. A., \& Oakes, L. M. (2010). The influence of pets on infants' processing of cat and dog images. Infant Behavior and Development, 33(4), 619-628. https://doi.org/10.1016/j.infbeh.2010.07.015

Inagaki, K., \& Hatano, G. (1996). Young children's recognition of commonalities between animals and plants. Child Development, 67(6), 2823-2840.

Isherwood, Z. J., Schira, M. M., \& Spehar, B. (2017). The tuning of human visual cortex to variations in the $1 / \mathrm{f} \alpha$ amplitude spectra and fractal properties of synthetic noise images. NeuroImage, 146, 642-657. https://doi.org/10.1016/j.neuroimage.2016.10.013

Jeschonek, S., Marinovic, V., Hoehl, S., Elsner, B., \& Pauen, S. (2010). Do animals and furniture items elicit different brain responses in human infants? Brain and Development, 32(10), 863-871.

Jozwik, K. M., Kriegeskorte, N., \& Mur, M. (2016). Visual features as stepping stones toward semantics: Explaining object similarity in IT and perception with non-negative least squares. Neuropsychologia, 83, 201-226.

Julesz, B. (1981). A theory of preattentive texture discrimination based on first-order statistics of textons. Biological Cybernetics, 41(2), 131-138.

Kastner, S., Weerd, P. D., \& Ungerleider, L. G. (2000). Texture Segregation in the Human Visual Cortex: A Functional MRI Study. Journal of Neurophysiology, 83(4), 2453-2457.

Kavšek, M. J., Yonas, A., \& Granrud, C. E. (2012). Infants' sensitivity to pictorial depth cues: A review and meta-analysis of looking studies. Infant Behavior and Development, 35(1), 109-128. https://doi.org/10.1016/j.infbeh.2011.08.003

Kayser, C., Körding, K. P., \& König, P. (2004). Processing of complex stimuli and natural scenes in the visual cortex. Current Opinion in Neurobiology, 14(4), 468-473.

Keil, F. C., Greif, M. L., \& Kerner, R. S. (2007). A world apart: How concepts of the constructed world are different in representation and in development. Creations of the Mind: Theories of Artifacts and Their Representation, 231-245.

Kellman, P. J. (2001). Separating processes in object perception. Journal of Experimental Child Psychology, 78(1), 84-97.

Kellman, P. J., \& Arterberry, M. E. (2007). Infant Visual Perception. In Handbook of Child Psychology. John Wiley \& Sons, Inc. https://doi.org/10.1002/9780470147658.chpsy0203

Kellman, P. J., \& Shipley, T. F. (1991). A theory of visual interpolation in object perception. Cognitive Psychology, 23(2), 141-221.

Kim, J., \& Anderson, B. L. (2010). Image statistics and the perception of surface gloss and lightness. 
Journal of Vision, 10(9), 3-3.

Kimchi, R. (2015). The perception of hierarchical structure. Oxford Handbook of Perceptual Organization, 129-149.

Knill, D. C., Field, D., \& Kerstent, D. (1990). Human discrimination of fractal images. JOSA A, 7(6), 1113-1123. https://doi.org/10.1364/JOSAA.7.001113

Knyazeva, M. G. (2013). Splenium of corpus callosum: Patterns of interhemispheric interaction in children and adults. Neural Plasticity, 2013.

Köster, M., Castel, J., Gruber, T., \& Kärtner, J. (2017). Visual cortical networks align with behavioral measures of context-sensitivity in early childhood. NeuroImage, 163, 413-418. https://doi.org/10.1016/j.neuroimage.2017.08.008

Köster, M., Kayhan, E., Langeloh, M., \& Hoehl, S. (2020). Making Sense of the World: Infant Learning From a Predictive Processing Perspective. Perspectives on Psychological Science, 15(3), 562-571. https://doi.org/10.1177/1745691619895071

Kovács, I. (2000). Human development of perceptual organization. Vision Research, 40(10-12), $1301-1310$.

Läge, D., Egli, S., Riedel, M., Strauss, A., \& Möller, H.-J. (2011). Combining the categorical and the dimensional perspective in a diagnostic map of psychotic disorders. European Archives of Psychiatry and Clinical Neuroscience, 261(1), 3-10. https://doi.org/10.1007/s00406-010-0125-y

Landy, M., \& Graham, N. (2004). Visual perception of texture. In The visual neurociences (pp. 11061118). MIT Press.

Lange-Küttner, C., Kerzmann, A., \& Heckhausen, J. (2002). The emergence of visually realistic contour in the drawing of the human figure. British Journal of Developmental Psychology, 20(3), 439-463. https://doi.org/10.1348/026151002320620415

Lawrence, M. A. (2016). ez: Easy Analysis and Visualization of Factorial Experiments (4.4-0). https://CRAN.R-project.org/package=ez

Leat, S. J., Yadav, N. K., \& Irving, E. L. (2009). Development of Visual Acuity and Contrast Sensitivity in Children. Journal of Optometry, 2(1), 19-26. https://doi.org/10.3921/joptom.2009.19

LoBue, V., \& Adolph, K. E. (2019). Fear in infancy: Lessons from snakes, spiders, heights, and strangers. Developmental Psychology, 55(9), 1889-1907. https://doi.org/10.1037/dev0000675

LoBue, V., \& DeLoache, J. S. (2008). Detecting the Snake in the Grass: Attention to Fear-Relevant Stimuli by Adults and Young Children. Psychological Science, 19(3), 284-289. https://doi.org/10.1111/j.1467-9280.2008.02081.x

LoBue, V., \& Rakison, D. H. (2013). What we fear most: A developmental advantage for threatrelevant stimuli. Developmental Review, 33(4), 285-303.

Long, B., Störmer, V. S., \& Alvarez, G. A. (2017). Mid-level perceptual features contain early cues to animacy. Journal of Vision, 17(6), 20-20. https://doi.org/10.1167/17.6.20

Madole, K. L., \& Oakes, L. M. (1999). Making sense of infant categorization: Stable processes and changing representations. Developmental Review, 19(2), 263-296. 
Mair, P., Borg, I., \& Rusch, T. (2016). Goodness-of-Fit Assessment in Multidimensional Scaling and Unfolding. Multivariate Behavioral Research, 51(6), 772-789. https://doi.org/10.1080/00273171.2016.1235966

Malt, B. C., \& Sloman, S. A. (2007). Artifact categorization: The good, the bad, and the ugly. Creations of the Mind: Theories of Artifacts and Their Representation, 85-123.

Mandler, J. M., \& McDonough, L. (1998). On developing a knowledge base in infancy. Developmental Psychology, 34(6), 1274.

Markman, E. M. (1989). Categorization and naming in children: Problems of induction. Mit Press.

Marr, D. (1976). Early processing of visual information. Philosophical Transactions of the Royal Society of London. B, Biological Sciences, 275(942), 483-519.

Matan, A., \& Carey, S. (2001). Developmental changes within the core of artifact concepts. Cognition, $78(1), 1-26$.

Maurer, D., \& Lewis, T. L. (2013). Sensitive Periods in Visual Development. The Oxford Handbook of Developmental Psychology, Vol. 1. https://doi.org/10.1093/oxfordhb/9780199958450.013.0008

Murtagh, F., \& Legendre, P. (2011). Ward's hierarchical clustering method: Clustering criterion and agglomerative algorithm. ArXiv Preprint ArXiv:1111.6285.

Nardini, M., Bedford, R., \& Mareschal, D. (2010). Fusion of visual cues is not mandatory in children. Proceedings of the National Academy of Sciences, 107(39), 17041-17046.

Nassi, J. J., \& Callaway, E. M. (2009). Parallel Processing Strategies of the Primate Visual System. Nature Reviews. Neuroscience, 10(5), 360-372. https://doi.org/10.1038/nrn2619

Nazzi, T., \& Gopnik, A. (2001). Linguistic and cognitive abilities in infancy: When does language become a tool for categorization? Cognition, 80(3), B11-B20.

New, J., Cosmides, L., \& Tooby, J. (2007). Category-specific attention for animals reflects ancestral priorities, not expertise. Proceedings of the National Academy of Sciences, 104(42), 16598-16603.

Nguyen, S. P., \& Gelman, S. A. (2002). Four and 6-year olds' biological concept of death: The case of plants. British Journal of Developmental Psychology, 20(4), 495-513.

Nieuwenhuis, R., Te Grotenhuis, H. F., \& Pelzer, B. J. (2012). Influence. ME: tools for detecting influential data in mixed effects models.

Nosofsky, R. M., Sanders, C. A., \& McDaniel, M. A. (2018). Tests of an exemplar-memory model of classification learning in a high-dimensional natural-science category domain. Journal of Experimental Psychology: General, 147(3), 328-353. https://doi.org/10.1037/xge0000369

Nosofsky, R. M., Sanders, C. A., Meagher, B. J., \& Douglas, B. J. (2017). Toward the development of a feature-space representation for a complex natural category domain. Behavior Research Methods, $1-27$.

Opfer, J. E. (2002). Identifying living and sentient kinds from dynamic information: The case of goaldirected versus aimless autonomous movement in conceptual change. Cognition, 86(2), 97-122.

Opfer, J. E., \& Gelman, S. A. (2011). Development of the animate-inanimate distinction. In U. C. Goswami (Ed.), The Wiley-Blackwell handbook of childhood cognitive development (Vol. 2, pp. 
213-238). Blackwell Publishers Ltd.

Oudeyer, P.-Y., \& Smith, L. B. (2016). How Evolution May Work Through Curiosity-Driven Developmental Process. Topics in Cognitive Science, 8(2), 492-502. https://doi.org/10.1111/tops.12196

Panis, S., De Winter, J., Vandekerckhove, J., \& Wagemans, J. (2008). Identification of everyday objects on the basis of fragmented outline versions. Perception, 37(2), 271-289.

Pauen, S., \& Hoehl, S. (2015). Preparedness to Learn About the World: Evidence from Infant Research. In T. Breyer (Ed.), Epistemological Dimensions of Evolutionary Psychology (pp. 159173). Springer New York. https://doi.org/10.1007/978-1-4939-1387-9_8

Pelz, J. B., \& Rothkopf, C. (2007). Oculomotor behavior in natural and man-made environments. In R. P. G. Van Gompel, M. H. Fischer, W. S. Murray, \& R. L. Hill (Eds.), Eye Movements (pp. 661676). Elsevier.

Perrinet, L. U., \& Bednar, J. A. (2015). Edge co-occurrences can account for rapid categorization of natural versus animal images. Scientific Reports, 5, 11400.

Pomaranski, K. I., Hayes, T. R., Kwon, M.-K., Henderson, J. M., \& Oakes, L. M. (2021). Developmental changes in natural scene viewing in infancy. Developmental Psychology, 57(7), 1025 .

Portilla, J., \& Simoncelli, E. P. (2000). A Parametric Texture Model Based on Joint Statistics of Complex Wavelet Coefficients. International Journal of Computer Vision, 40(1), 49-70. https://doi.org/10.1023/A:1026553619983

Quinn, P. C. (2011). Born to categorize. In U. C. Goswami (Ed.), The Wiley-Blackwell handbook of childhood cognitive development (2nd ed., pp. 129-152). Blackwell Publishers Ltd.

R Core Team. (2019). R: A language and environment for statistical computing. R Foundation for Statistical Computing. https://www.R-project.org/

Rakison, D. H., \& Derringer, J. (2008). Do infants possess an evolved spider-detection mechanism? Cognition, 107(1), 381-393. https://doi.org/10.1016/j.cognition.2007.07.022

Rakison, D. H., \& Poulin-Dubois, D. (2001). Developmental origin of the animate-inanimate distinction. Psychological Bulletin, 127(2), 209.

Rakison, D. H., \& Yermolayeva, Y. (2010a). Infant categorization. WIREs Cognitive Science, 1(6), 894-905. https://doi.org/10.1002/wcs.81

Rakison, D. H., \& Yermolayeva, Y. (2010b). Infant categorization. Wiley Interdisciplinary Reviews: Cognitive Science, 1(6), 894-905.

Rao, A. R., \& Lohse, G. L. (1993). Identifying high level features of texture perception. CVGIP: Graphical Models and Image Processing, 55(3), 218-233.

Rao, A. R., \& Lohse, G. L. (1996). Towards a texture naming system: Identifying relevant dimensions of texture. Vision Research, 36(11), 1649-1669.

Read, D., \& Van Der Leeuw, S. (2008). Biology is only part of the story.... Philosophical Transactions of the Royal Society B: Biological Sciences, 363(1499), 1959-1968. 
Redies, C., Hasenstein, J., \& Denzler, J. (2007). Fractal-like image statistics in visual art: Similarity to natural scenes. Spatial Vision, 21(1-2), 137-148.

Renninger, L. W., \& Malik, J. (2004). When is scene identification just texture recognition? Vision Research, 44(19), 2301-2311.

Revelle, W. (2018). psych: Procedures for Psychological, Psychometric, and Personality Research (1.8.10) [R]. Northwestern University. https://CRAN.R-project.org/package=psych

Rhodes, M., \& Gelman, S. A. (2009). A developmental examination of the conceptual structure of animal, artifact, and human social categories across two cultural contexts. Cognitive Psychology, 59(3), 244-274. https://doi.org/10.1016/j.cogpsych.2009.05.001

Rosch, E., Mervis, C. B., Gray, W. D., Johnson, D. M., \& Boyes-Braem, P. (1976). Basic objects in natural categories. Cognitive Psychology, 8(3), 382-439.

Ruderman, D. L. (1997). Origins of scaling in natural images. Vision Research, 37(23), 3385-3398.

Scherf, K. S., Behrmann, M., Kimchi, R., \& Luna, B. (2009). Emergence of global shape processing continues through adolescence. Child Development, 80(1), 162-177.

Schlegelmilch, K., \& Wertz, A. E. (2022). Visual segmentation of complex naturalistic structures in an infant eye-tracking search task. PsyArXiv. https://doi.org/10.31234/osf.io/6ey8f

Schmidt, F., Hegele, M., \& Fleming, R. W. (2017). Perceiving animacy from shape. Journal of Vision, 17(11), 10-10. https://doi.org/10.1167/17.11.10

Schmuckler, M. A. (1993). Perception-action coupling in infancy. In Advances in psychology (Vol. 97, pp. 137-173). Elsevier.

Şerban, P., Wilson, J. R. U., Vamosi, J. C., \& Richardson, D. M. (2008). Plant Diversity in the Human Diet: Weak Phylogenetic Signal Indicates Breadth. BioScience, 58(2), 151-159. https://doi.org/10.1641/B580209

Shepard, R. N. (1992). The perceptual organization of colors: An adaptation to regularities of the terrestrial world? In The adapted mind: Evolutionary psychology and the generation of culture. (pp. 495-532). Oxford University Press.

Sireteanu, R., Encke, I., \& Bachert, I. (2005). Saliency and context play a role in infants' texture segmentation. Vision Research, 45(16), 2161-2176. https://doi.org/10.1016/j.visres.2005.02.003

Siu, C., \& Murphy, K. (2018). The development of human visual cortex and clinical implications. Eye and Brain, Volume 10, 25-36. https://doi.org/10.2147/EB.S130893

Skoczenski, A. M., \& Norcia, A. M. (2002). Late maturation of visual hyperacuity. Psychological Science, 13(6), 537-541.

Sloutsky, V. M. (2003). The role of similarity in the development of categorization. Trends in Cognitive Sciences, 7(6), 246-251.

Sloutsky, V. M., \& Fisher, A. V. (2004). Induction and categorization in young children: A similaritybased model. Journal of Experimental Psychology: General, 133(2), 166.

Smith, L. B. (1979). Perceptual development and category generalization. Child Development, 705715 . 
Soja, N. N., Carey, S., \& Spelke, E. S. (1991). Ontological categories guide young children's inductions of word meaning: Object terms and substance terms. Cognition, 38(2), 179-211.

Taylor, G., Hipp, D., Moser, A., Dickerson, K., \& Gerhardstein, P. (2014). The development of contour processing: Evidence from physiology and psychophysics. Frontiers in Psychology, 5. https://doi.org/10.3389/fpsyg.2014.00719

Torralba, A., \& Oliva, A. (2003). Statistics of natural image categories. Network: Computation in Neural Systems, 14(3), 391-412.

Truxaw, D., Krasnow, M. M., Woods, C., \& German, T. P. (2006). Conditions under which function information attenuates name extension via shape. Psychological Science, 17(5), 367-371.

Tversky, A. (1977). Features of similarity. Psychological Review, 84(4), 327.

van den Boomen, C., Lamme, V. A. F., \& Kemner, C. (2014). Parallel development of ERP and behavioural measurements of visual segmentation. Developmental Science, 17(1), 1-10. https://doi.org/10.1111/desc.12093

Wagemans, J., Elder, J. H., Kubovy, M., Palmer, S. E., Peterson, M. A., Singh, M., \& von der Heydt, R. (2012). A century of Gestalt psychology in visual perception: I. Perceptual grouping and figureground organization. Psychological Bulletin, 138(6), 1172.

Wallis, T. S. A., Funke, C. M., Ecker, A. S., Gatys, L. A., Wichmann, F. A., \& Bethge, M. (2017). A parametric texture model based on deep convolutional features closely matches texture appearance for humans. Journal of Vision, 17(12), 5. https://doi.org/10.1167/17.12.5

Ward, J. H. (1963). Hierarchical grouping to optimize an objective function. Journal of the American Statistical Association, 58(301), 236-244.

Warrington, E. K., \& McCarthy, R. A. (1987). Categories of knowledge: Further fractionations and an attempted integration. Brain, 110(5), 1273-1296.

Wertz, A. E. (2019). How plants shape the mind. Trends in Cognitive Sciences, 23(7), 528-531.

Wertz, A. E., \& Wynn, K. (2014a). Thyme to touch: Infants possess strategies that protect them from dangers posed by plants. Cognition, 130(1), 44-49. https://doi.org/10.1016/j.cognition.2013.09.002

Wertz, A. E., \& Wynn, K. (2014b). Selective Social Learning of Plant Edibility in 6- and 18-MonthOld Infants. Psychological Science, 25(4), 874-882. https://doi.org/10.1177/0956797613516145

Wertz, A. E., \& Wynn, K. (2019). Can I eat that too? 18-month-olds generalize social information about edibility to similar looking plants. Appetite, 138, 127-135.

https://doi.org/10.1016/j.appet.2019.02.013

Westermann, G., \& Mareschal, D. (2012). Mechanisms of developmental change in infant categorization. Cognitive Development, 27(4), 367-382.

https://doi.org/10.1016/j.cogdev.2012.08.004

Wickens, T. D. (2002). Elementary signal detection theory. Oxford University Press, USA.

Younger, B., \& Gotlieb, S. (1988). Development of categorization skills: Changes in the nature or structure of infant form categories? Developmental Psychology, 24(5), 611.

Zachariou, V., Del Giacco, A. C., Ungerleider, L. G., \& Yue, X. (2018). Bottom-up processing of 
curvilinear visual features is sufficient for animate/inanimate object categorization. Journal of Vision, 18(12), 3-3.

Zhu, S.-C., Guo, C., Wang, Y., \& Xu, Z. (2005). What are Textons? International Journal of Computer Vision, 62(1-2), 121-143. https://doi.org/10.1023/B:VISI.0000046592.70770.61

Zujovic, J., Pappas, T. N., \& Neuhoff, D. L. (2013). Structural texture similarity metrics for image analysis and retrieval. IEEE Transactions on Image Processing, 22(7), 2545-2558. 